\title{
Deep Learning Based COVID-19 Detection: Challenges and
} Future Directions

This paper was downloaded from TechRxiv (https://www.techrxiv.org).

\section{LICENSE}

CC BY 4.0

SUBMISSION DATE / POSTED DATE

$20-05-2021 / 25-05-2021$

\section{CITATION}

Khan, Muhammad Khurram; Arshad, Qurat-ul-Ain; Azam, Faisal; Khan, Wazir Zada (2021): Deep Learning Based COVID-19 Detection: Challenges and Future Directions. TechRxiv. Preprint. https://doi.org/10.36227/techrxiv.14625885

$\mathrm{DOI}$

10.36227/techrxiv.14625885 


\title{
Deep Learning Based COVID-19 Detection: Challenges and Future Directions
}

\author{
Qurat-ul-Ain Arshad, Wazir Zada Khan*, Faisal Azam, Muhammad Khurram Khan*
}

\begin{abstract}
Coronavirus (COVID-19) is an ecumenical pandemic that has affected the whole world drastically by raising a global calamitous situation. Due to this pernicious disease, millions of people have lost their lives. The scientists are still far from knowing how to tackle the coronavirus due to its multiple mutations found around the globe. Standard testing technique called Polymerase Chain Reaction (PCR) for the clinical diagnosis of COVID-19 is expensive and time consuming. However, to assist specialists and radiologists in COVID-19 detection and diagnosis, deep learning plays an important role. Many research efforts have been done that leverage deep learning techniques and technologies for the identification or categorization of COVID-19 positive patients, and these techniques are proved to be a powerful tool that can automatically detect or diagnose COVID-19 cases. In this paper, we identify significant challenges regarding deep learning-based systems and techniques that use different medical imaging modalities, including Cough and Breadth, Chest X-ray, and Computer Tomography (CT) to combat COVID-19 outbreak. We also pinpoint important research questions for each category of challenges. The challenges highlighted in this paper will call an attention to the noticeable weaknesses and problems in the existing deep learning based COVID-19 detection systems and techniques. Moreover, the research questions for each challenge will guide the researchers to come up with novel solutions in COVID-19 detection.
\end{abstract}

Index Terms-COVID-19, Coronavirus, SARS-CoV-2, Detection, Diagnosis, CT-Scan, X-Ray, Sound Analysis, Deep Learning.

\section{INTRODUCTION}

Today the world is facing a humanitarian emergency in the form of a virus called Severe Acute Respiratory Syndrome Coronavirus 2 (SARS-CoV-2), which was announced to be renamed as Coronavirus Disease-2019 (COVID-19) by World Health Organization (WHO) [1]. The WHO confirmed it as an epidemic on 11 March 2020 [2]. It was initially started in Wuhan in China's Hubei Province in early December 2019 and that resulted in a cluster of viral pneumonia cases [3]. As per the available statistics, due to this virus an escalating number of reported infections were caused in humans in China and globally, gaining entry to over 210 countries and territories [4]. It has caused illnesses ranging from the common cold to acute respiratory tract infection, which has led to death in case of much severity. A plethora of viral respiratory diseases can affect the human pulmonary system through nose, mouth, eyes and air borne transmission of contact-based inoculation of infectious material in droplets. A number of viral respiratory infections with pandemic potential have been emerged in the past decades that have challenged humans including the Severe Acute Respiratory Syndrome coronavirus (SARSCoV) which emerged in China in 2002 [1], swine-origin pandemic (H1N1) influenza virus, which emerged in Mexico in 2009 [5] and the Middle East Respiratory Syndrome

- Q. Arshad and F. Azam are with Department of Computer Science, CUI, Islamabad, Pakistan. Email: brightsuccess_12@yahoo.com, faisal@cuiwah.edu.pk.

- W. Z. Khan is with Department of Computer Science, Capital University of Science and Technology, Islamabad, Pakitsan. E-mail: wazir.zada@cust.edu.pk $\left({ }^{*}\right.$ Corresponding author $)$

- M. K. Khan is with Center of Excellence in Information Assurance, King Saud University, Riyadh 11653, Saudi Arabia. E-mail: mkhurram@ksu.edu.sa. $\left({ }^{*}\right.$ Corresponding author) coronavirus (MERS-CoV), which emerged in Saudi Arabia in 2012 [6]. These three viruses frequently lead to lower respiratory tract disease with similar syndromes like fever and cough. It is suspected by the experts that COVID-19 virus is transmitted from animals to humans, but there are mixed reports on the origin of the virus [7]. Collectively experts and increasing evidences conclude that COVID-19 virus had a natural origin in bats, as with previous such respiratory viruses [8], [9], [10]. Naturally over time, all viruses mutate and SARS-CoV-2 is no exception. In the last winter, when the coronavirus SARS-CoV-2 burst upon the world, the scientists thought it was stable. But it was not like that and in recent months, several new variants of the original virus have been spotted. The worry of the time is that a rapid rise in COVID-19 cases all over the world have overwhelmed all healthcare systems. The mutations of coronaviruses are emerging with time and thus, there is a lack of general preventive vaccines for clinical use in humans against such viruses, making it a serious global threat.

Imminently, there is a rapid spread of novel COVID19 disease, putting even the best healthcare systems across the world under tremendous pressure. Some of the initial symptoms identified by WHO and CDC indicate plausible COVID-19 infection are mild which include dry cough, fever, diarrhoea, vomiting and myalgia which yet rapidly change into extremely critical condition of the patient and suffering from multi-organ failures may lead to even fatal outcomes. Thus, early treatment morbidity rates can be reduced by seeking the early treatment by detecting the disease at the earliest using the limited knowledge base and resources so that abrupt deteriorations can be reduced [11]. According to [12] it is observed that the regions with high temperatures and humidity have minimal spreading of 


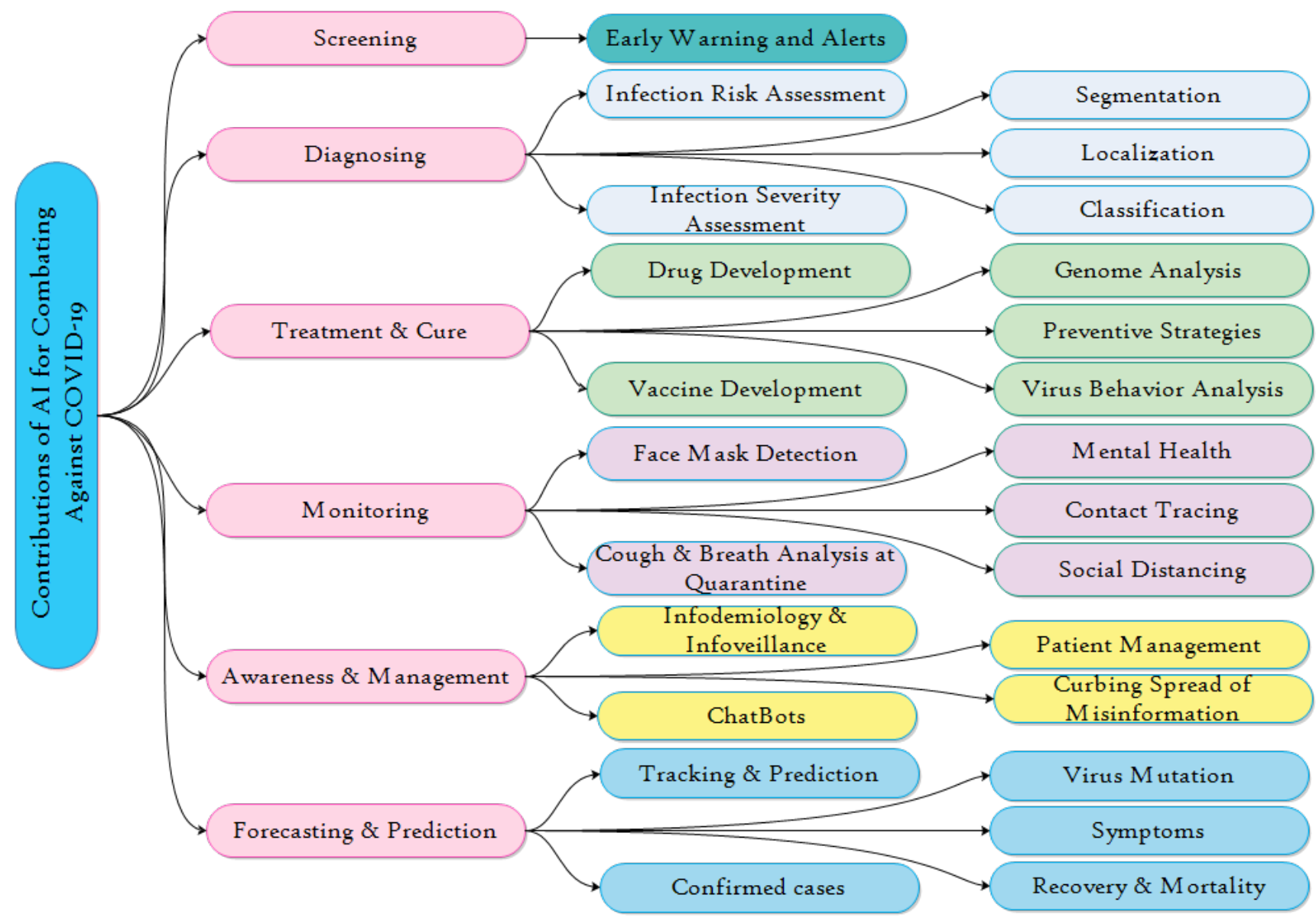

Fig. 1. Al contributions for Combating COVID-19

COVID-19. Another observation in [13] infers that COVID19 virus is dependent upon some environmental conditions such as water droplets, physical contact, and hygiene for its growth and spread. Although, according to WHO, specific medication is still not available for COVID-19 but since, the number of COVID-19 cases has been increasing at an accelerated rate, WHO has come up with some strategies to combat COVID-19. First strategy says that all the suspected cases should be tested and COVID-19 positive patients should be isolated and their contacts and traveling history should be observed. Another important strategy implies that a non-compromising lockdown should be followed and imposed so that social distancing is ensured for the containment of the disease.

The Real-Time Polymerase Chain Reaction (RT-PCR) is the most widely used traditional laboratory method for detecting the COVID-19 virus. In this test, a sample from person's nose or throat is collected where the COVID-19 virus gathers and the substances like fats and proteins are removed by using several chemicals and finally is extracted. If virus is present then this extracted RNA contains the person's own genetic material as well as virus's RNA. RT-PCR method for the detection of the COVID-19 virus is continued to be the most accurate available method since, is is specific and highly sensitive. The process of detecting COVID-19 through RT-PCR is carried out within a closed tube and thus has a lower potential for contamination or errors, deliver- ing a reliable diagnosis. Apart from these advantages this method has some limitations that are important to be noted [14], [15]. First, the instrument is too costly and kits are not available for all kinds of genes and disorders. This method requires specialised bio-containment laboratories, operated by highly trained technicians, thus, making it difficult to scale. Second, this technique will only indicate the presence of viral material during infection and will not indicate if a person was infected, has or has not developed immunity and subsequently recovered. Thirdly, due to the presence of amplification inhibitors, variable viral load in specimens or improper collection this testing method can have high false-negative rates, and thus, probable cases may remain negative. In addition, with evidence it happens many times that even after getting negative results, COVID-19 symptoms can be detected by radiological images of patients [16]. However, scientists and researchers are working hard for the enhancement and efficiency of COVID-19 diagnostic and detection systems by overcoming the limitations of RT-PCR testing.

The advancements and developments in AI can facilitate healthcare personnel with the improved capacity of imaging tools [17]. For the prognosis of various diseases and to identify and monitor them, the radiologists can make clinical decisions with the assistance of computer aided networks, which not only decrease the picture diagnostic time but also increase the accuracy and execution of the 
diagnosis. By leveraging AI techniques, the work performance can be enhanced through correct diagnostic precision in X-ray and CT imaging, which further makes it easier to test. AI and DL have been commonly used for effective disease identification, prediction and detection thus, results are enormous in disease classification, image identification, organ recognition and geographic infection classification e.g deep learning has been used in various medical systems such as in biomedicine [18], smart healthcare [19], [20], [21], [22], drug discovery [23], [24], [25], medical image analysis [26], [27], and last but not the least to successfully diagnose Pneumonia either from chest X-ray images or CT scans [28], [29], [30]. With the emergence of COVID-19, since, pneumonia and COVID-19 exhibit similar/overlapping symptoms and affect the human lungs, it has become more challenging for the researchers to distinguish between the chest X-ray images of pneumonia patients and COVID-19 patients. In the current health crisis and Coronavirus pandemic, health industry, practitioners and researchers are utilizing Artificial Intelligence (AI) for tracking and controlling its growth and spread. Figure 1 demonstrates a number of AI contributions in fighting against COVID-19 and to curtail its adverse effects. Moreover, the researchers are eagerly looking for new techniques and technologies based on deep learning for tracking the speed and detecting the growth rate of the Coronavirus, and identifying the risk and severity of patients. Moreover, using AI, previous patient data can be adequately analyzed to anticipate the possibility of death. There are three types of COVID-19 data, Clinical data, biomedical data, and online data.

There are some studies [11], [31], [32], [33], [34], [35], [36], [37] that have attempted to compile the literature related to COVID-19 detection but they all have contemplated and reviewed only one facet, either detection through CT scans or X-ray images or cough analysis and also they fail to cover the recent literature comprehensively. Moreover, these studies lack to cover important challenges and research questions in this field. The main objective of this research is to identify significant challenges and research questions in detecting COVID-19 through deep learning. Our contributions are threefold:

- First we describe in detail the general pipeline of image processing for COVID-19 detection.

- Second we describe in detail the deep learning based detection of COVID-19 using different kinds of modalities.

- Third, we identify, categorize and present some important challenges in detecting COVID-19 through deep learning. The research questions for each category of challenges are also identified.

The rest of the paper is organized as follows: Section 2 presents the general procedure followed for COVID-19 detection in image processing field using DL. Section 3 presents a taxonomy of modalities used for deep learning based techniques for COVID-19 detection. Section 4 presents a taxonomy of significant challenges in detecting COVID-19 using DL and research questions for each category. Section 5 finally concludes the paper.

\section{General Pipeline of Image processing FOR COVID-19 DETECTION USING DL}

In the automated diagnosis of COVID-19 in patients, DL is extensively used. Generally, several steps are followed by deep learning based COVID-19 detection systems including Data collection, Data pre-processing, Segmentation, Localization, Feature Extraction, Feature Selection and Classification. The deep learning based COVID-19 diagnosis systems follow the general pipeline of as illustrated in Fig 2.

\subsection{Image Acquisition}

In the image acquisition stage, radiography images (CT scans or $\mathrm{X}$ rays images) or Sound data of the patient (speech, cough and breadth) are collected using different technologies and machines. CT scans range from the apex to the lung base and during a single breath hold they are acquired. CT images are reconstructed from the acquired raw data and then for subsequent readings and diagnosis they are transmitted through picture archiving communications systems (PACS). An inevitable contact between the technicians and patients is required in the conventional imaging workflow e.g. in positioning the patients, technicians assist in posing the patients but to avoid the severe risks of infection in this pandemic of COVID-19, it is important to employ a contactless and automated image acquisition workflow. Sound data such as breathing, cough and speech can also be collected using smartphone sensors or wearable sensors and can be utilized for COVID-19 diagnosis.

\subsection{Pre-Processing}

The primary task of pre-processing is to emphasize aspects of image and improve the quality of the raw images to make it in a form that is well suited for further processing by machine vision system and help in recognition tasks or even in deep learning training phase. In pre-processing certain parameters of images are improved such as smoothing the inner part of the region, improving the signal-to noise ratio and removing the irrelevant noise and undesired parts in the background, enhancing the visual appearance of CT images image, and preserving its edges [38]. Since CXR images and CT scan images are collected from different sources, to ensure uniformity across different datasets, they are resized and transformed from RGB to grayscale. To speed up the convergence performance, the resultant images are then subjected to min-max normalization. In case of speech data recorded in the installed applications and converted into a digital format, unwanted components such as pauses, background noise and stammering are also removed from the digital signals in the pre-processing phase. Filtering and general signal processing techniques are applied to clear the areas to be processed for voice activity detection phase. After obtaining the actual signal, feature selection algorithms are applied to categorize the input signal into a specific characterized speech signal [39].

\subsection{Segmentation}

Segmentation is the process of dividing or partitioning a digital image into multiple segments. By diving the image 
into segments, important segments can be picked for processing the image. For COVID-19 detection, the lung part in a chest $X$-ray images is examined. For a successful detection, lung part in each image should be segmented. Moreover, In fighting against COVID-19, important information can be deducted by qualitataively evaluating and delination of lung infections and longitudina changes in CT scans of COVID-19 patients. It is indicated that the distinctive infection indication of ground-glass opacity (GGO) and consolidation can be detected through segmentation of CT scans of COVID-19 patients [40]. It is laborious, tedious and time consuming to manually project the lung infections with the dependence of the accuracy of infection annotation heavily on the knowledge and experience of the radiologist and often influenced by individual bias and clinical experiences as it is a subjective task. Thus, the automatic and accurate segmentation techniques can provide rapid screening of COVID-19. DL based techniques for COVID-19 detection have adopt different types of segmentation techniques such as few shot segmentation [41] and semantic segmentation [42].

Some of the DL based techniques are designed for severity assessment of COVID-19 patients by leveraging segmentation of lung or lung lobe in CT images as a prerequisite procedure for diagnosis purposes [43], [44], [45], [46]. However, in most of these methods the lung lobe segmentation and disease diagnosis are treated as two separate tasks and their underlying correlation is ignored which ultimately slows down the classification and detection or prediction process. Thus, it is instinctive that lung lobe segmentation and severity assessment/prediction are performed jointly in which there is no need to detect and crop the lung field and prediction performance is improved through faster learning [47], [48].

\subsection{Feature Extraction}

Feature Extraction is an essential step towards classification because useful characteristics of the images can be provided by extracted features. Features can be of two types; handcrafted features (based on the texture, geometry, and morphological characteristics of the lung) and features obtained though transfer learning. The traditional machine learning methods for image recognition such as Bayesian networks (BNs), support vector machine (SVM), artificial neural networks (ANNs), k-nearest neighbors $(\mathrm{kNN})$ require these handcrafted features but they have a limitation that their accuracy is dependent upon the feature extraction step thus, making it difficult to find the most relevant feature which are needed to obtain the most effective result [49].The features obtained through transfer learning are the features that a trained ML model such as CNN identify and extract for some specific classification task. These ML models search for what features are best to classify the images. The diagnostic models [50], [51], [52] have used hand engineered features for COVID-19 detection whereas the models [53], [54], [55], have used off-the-shelf networks for feature extraction.

In feature extraction an initial set of the raw data is divided and reduced to more meaningful groups. It aims to reduce the number of features in a dataset by creating new features from the existing ones and then discarding the original features. Feature extraction is useful when dataset is large. In case of CT scan and X ray images, deep neural networks (DNN) can provide extraordinary capabilities for feature extraction from a large scale dataset. Deep learning algorithms such as CNN (which is widely used in COVID19 detection for features extraction) can be used to segment regions of interest (ROI) and capture fine structures in chest CT images and then self learned features can be easily extracted. In a study [56], the authors have developed a 3-dimensional convolutional neural network $(\mathrm{CNN})$ based framework which differentiated COVID-19 from the community acquired pneumonia by extracting features from $\mathrm{CT}$ scan images.

In a study [57], the authors have differentiated COVID19 from normal cases by using several pre-trained networks including DenseNet, MobileNet, ResNet, InceptionV3, Xception, InceptionResNetV2 for feature extraction of images. Then, six machine learning algorithms, including random forest, decision tree, AdaBoost, XGBoost, LightGBM and Bagging were used for training the extracted features. In another system proposed in [58] the authors have extracted features form CT images by using ResNet50 and then applied CNN for the classification. Moreover, in [59] a pipeline and multiview representation learning technique is proposed to classify COVID-19 using different types of extracted features from CT images. In [60] the authors have proposed a model in which random forest is used to automatically extract a number of features from $\mathrm{CT}$ images such infected lesion number, volume, surface area and histogram distribution.

In case of cough sounds dataset, cough features can be extracted such as wet cough and dry cough for COVID19 detection. However, it is time consuming to develop a classification model from a dataset with high dimentionality which may also converge to a local minima due to the large search space. Also, cough features of COVID-19 may overlap with other diseases so performance and accuracy can be immensely improved if a reduced set of relevant features form an audio sample are selected. It is thus, challenging to extract cough features however some attempts have been made by some researchers. The authors in [61] have collected and investigated both cough and breathing sound that can help in COVID-19 diagnosis. They have provided handcrafted features for cough and breathing sounds such as onset, duration, period, tempo, frequency,Mel-frequency cepstrum (MFCC), delta MFCC and delta delta MFCC, root mean square (RMS) energy, spectral centroid, roll-off and zero-crossing.

\subsection{Classification}

In deep learning (DL) mainly focues on on image's features extraction and classification in an automated manner [15], [62], [63], [64], [65], [66], [67], [68], [69], [70], [71]. By using Chest X-ray images (CXR/CRI) of patients, most researchers focused on DL techniques to detect COVID-19. Some of the researchers attempted to detect COVID from Non-COVID called which is called binary classification [72], [73], [74], [75], [76], [77]. Some of the researchers have proposed DL based techniques for the severity assessment of COVID19 patients. In these techniques binary classification problem is formulated for the the task of severity assessment 


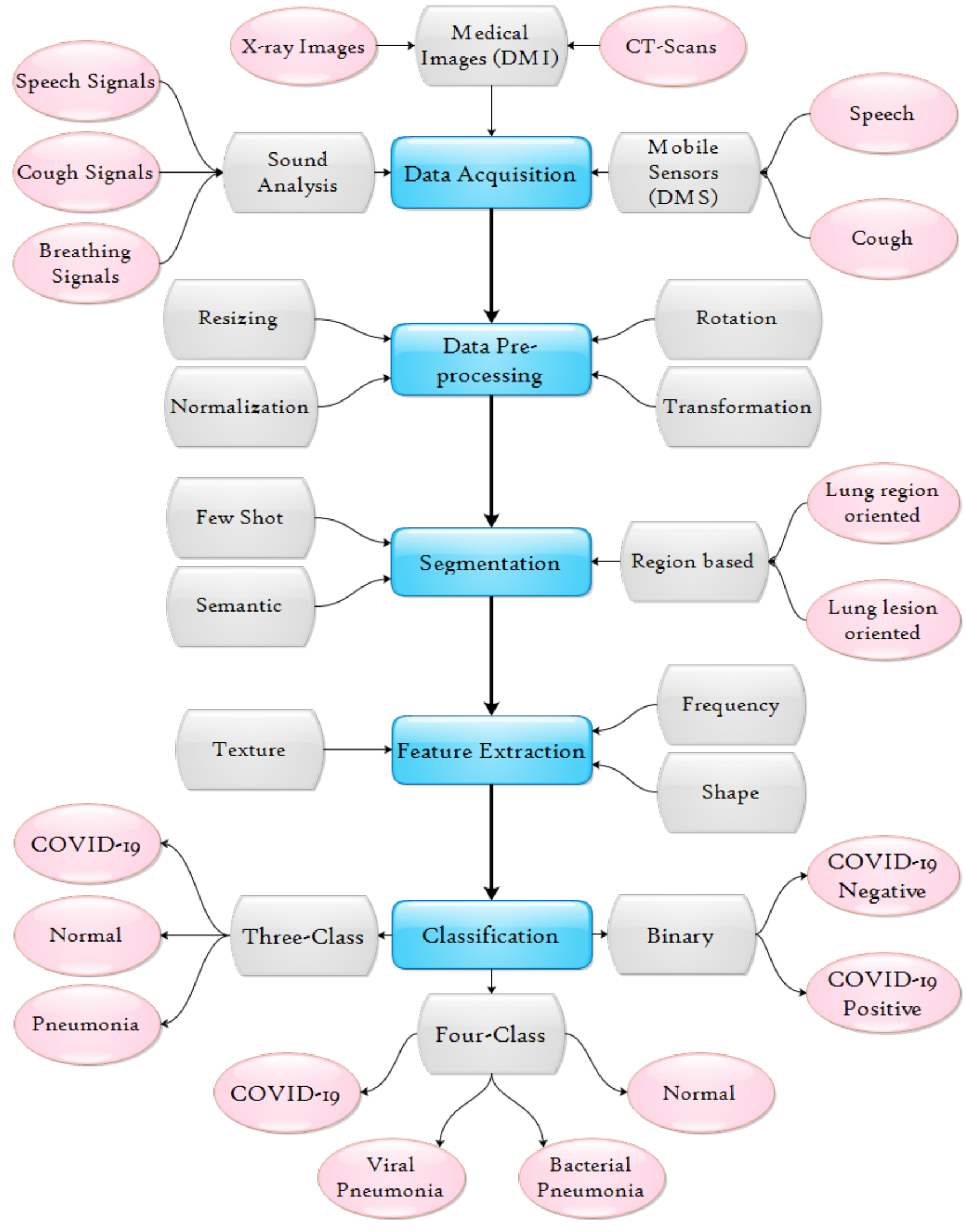

Fig. 2. Life-cycle of DL Based COVID-19 Detection

and finally a classification layer utilizes learned volume features for the severity assessment of COVID-19 patients i.e., severe or non-severe COVID-19 patients [47]. Others concerned with three class classification (COVID vs Normal 
vs pneumonia) [15], [62], [63], [64], [65], [66], [67], [68], [69], [78]. Only a few of the researchers focused on COVID-19 detection using four class classification (COVID vs Normal vs Bacterial pneumonia vs Viral pneumonia) [70], [71].

\section{Modalities used for COVID-19 Detection Through DeEp Learning}

In this section we devise a taxonomy for the modalities used by the research community for COVID-19 detection. The three types of modalities are Medical images, Sound and Smartphone based data. The taxonomy is presented in Figure 3.

\subsection{COVID-19 Detection through Medical Images (DMI)}

In the past, to successfully diagnose Pneumonia either from chest X-rays or CT scans, artificial intelligence (AI) based techniques were employed [28], [29], [30]. Since, in emergency cases, it might not be feasible to manually checking every CT image. Thus, presently, in this pandemic, AI can analyze CT scans or X-Rays of COVID-19 patients in 10 s, comparing to manual interpreting of $\mathrm{CT}$ scan by the clinicians in 15 minutes, thereby, reducing the strain on clinicians [44]. AI and deep learning algorithms are capable of capturing fine structures in chest CT scan and for the segmentation of regions of interest (ROI). Deep learning algorithms and more recently CNN algorithm is found to be effective in identifying COVID-19 via image classification and can easily extract self learned features for COVID-19 diagnosis and detection while differentiating it from other lung diseases and community acquired pneumonia [56]. In this section we will address the innovations of deep learning based techniques for COVID-19 detection by using chest Xray and $\mathrm{CT}$ imaging in depth.

The chest computed tomography (CT) is a valued feature of the assessment of patients with respiratory complications, as well as a great utility for elective surgical procedure monitoring and neurological examinations [79], [80]. In a chest CT examination procedure, $\mathrm{X}$-rays are transmitted through the patient's chest, which are then reconstructed into high-resolution medical images after being detected by radiation detectors. At present, for the detection of COVID19 , Thoracic CT scan and chest CT scans are extensively used by the clinical institutes around the world and are explored to be effective and complementary screening tool alongside RT-PCR [81]. Even more, CT scan provides more standard and intuitive information as compared to the RTPCR test [82], [83], [84], [85]. On the basis of CT scans of COVID-19 patients, radiologists can make medical decisions by possibly determining how badly the lungs are compromised and how the illness of the individual progresses. To be adopted as a vital method for supporting the diagnosis and management of patients with COVID-19 infection, there are certain patterns to look out for in a Thoracic CT scans including air bronchograms, perilobular pattern, groundglass opacity (GGO), crazy paving and consolidation, and reverse halo [86]. More importantly there is a need to know which part of the CT lung is most affected by COVID and specific patterns need to be look upon including bilateral, peripheral, inter-lobular septa thickening, multifocal patchy consolidation, basal predominant ground-glass opacity (GGO) and crazy-paving pattern with a peripheral distribution which have been declared as the findings of COVID-19 infection at chest CT images [87]. Novel strategies and proposals are required for simple classification and identification of COVID severity locations (CSL) since, few studies [88], [89] are not automated strategies for COVID severity locations (CSL).

Although analysis of CT scans provide more useful, fater and reliable results in the COVID-19 classification and assessment but there are a few challenges to use CT scans for COVID-19 detection. First of all, it is challenging for radiologists in distinguishing COVID-19 infections from non-COVID-19 infections using chest CT [90], [91] because some early studies have shown that a number of potential indicators for COVID-19 infections that are present in chest CT images may also be present in non-COVID-19 infections [88], [92], [93], [94]. Second, CT takes longer for imaging. The CT imaging is highly costly and the CT scanners are not available in many underdeveloped countries. Moreover, due to high radiation in $\mathrm{CT}$ imaging, pregnant women and children may face health risks. Third, CT scanning equipment are not portable, and there is a need to sanitize the room and equipment between patients followed by a delay of at least an hour [95]. Otherwise, there is a danger of risk of exposing the persons under investigation (PUIs), hospital staff and other patients to the COVID-19 virus [96] and its transmission from a patient to others due to CT scan tunnel contamination [97].

In many medical and epidemiological cases, $X$-ray (CXR) imaging has played a great role due to its wider availability. They have the potential in the monitoring and examination of various lung diseases such as pneumonia, infiltration, tuberculosis, hernia and atelectasis and thus, they are recognized as a valuable medical tool for a wide variety of examinations and procedures. They are used for diagnosing disease and monitoring therapy painlessly and non-invasively. CXR imaging also supports medical and surgical treatment planning by guiding the medical personnel when they are inserting devices inside the body such as stents, or catheters etc. or when they are treating tumors or removing blockages or blood clots. Currently, in this pandemic of COVID-19, CXR images are found to be very helpful in testing and evaluating COVID-19 patients. When attributable symptoms of COVID-19 like fever, cough, or dyspnoea are presented by the patients, doctors perform chest $\mathrm{X}$-rays as they are cheaper and quick and the radiologists look for ground glass opacities (in case of COVID-19 usually the first radiological sign), with portions of the lungs appearing as a "hazy" shade of grey instead of being black with fine white lung markings for blood vessels [98]. In the studies for detecting COVID-19 the researchers have looked for predominant patterns in chest radiographs, such as peripheral opacities, interstitial opacities, airspace opacities, diffuse airspace opacities, lobar consolidation, craniocaudal gradient and bilateral diffuse reticular interstitial lung thickening and the presence of pleural effusions [99], [100].

Comparing to CT scan imaging, chest X-ray (CXR) imaging is more beneficial and effective for emergency cases and due to its less ionizing radiations, simplicity, portability (due to portable instrumentation), operational speed, and 


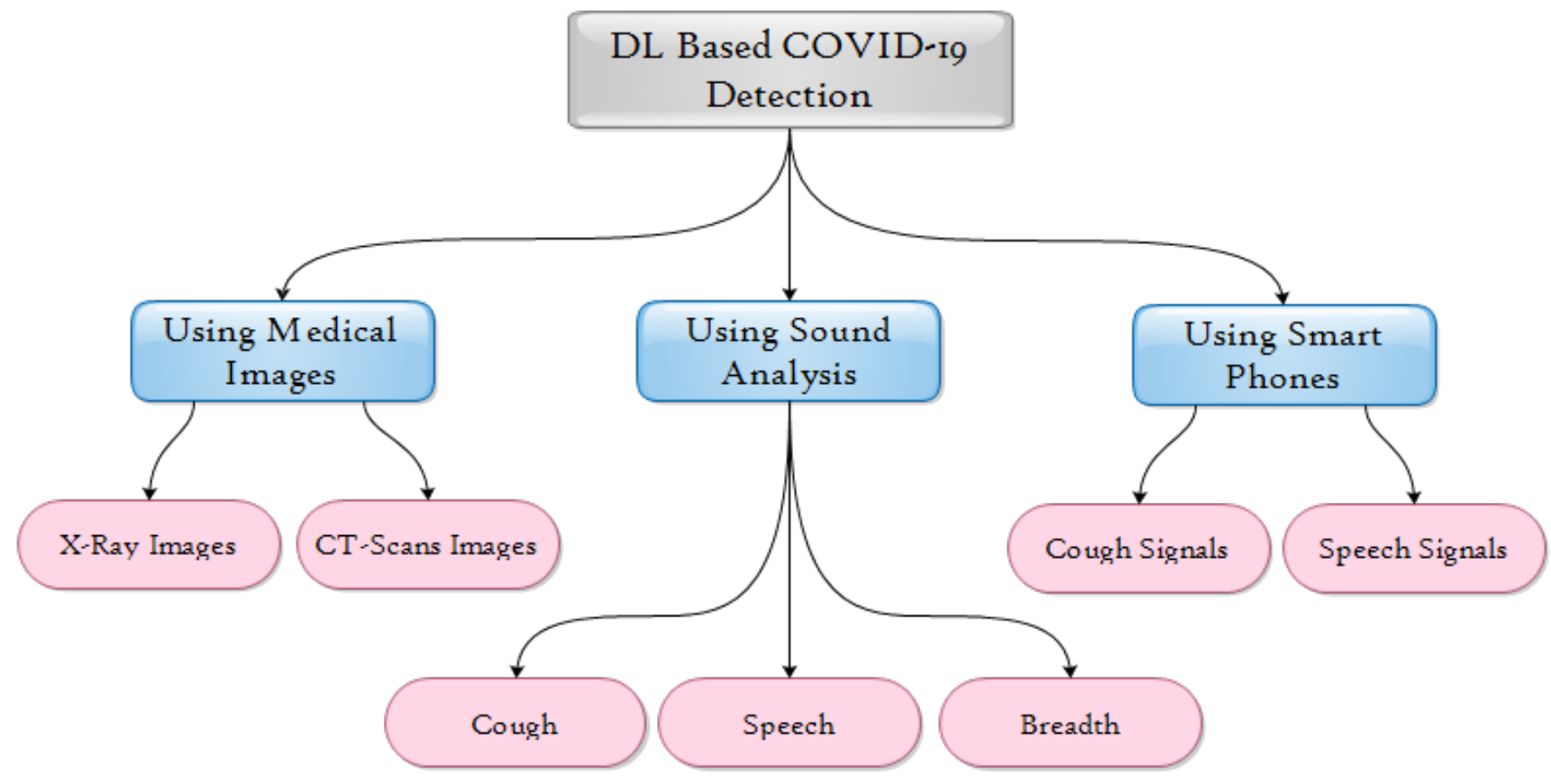

Fig. 3. Type of Modalities used for DL based COVID-19 Detection

low cost it is found to be promising. In CXR imaging, the personnel exposure is limited, and the PUIs can be imaged in more isolated rooms. Moreover, comparing to CT scan, obtaining CXR images, sanitation is much less complex [101]. Moreover, as CXR images are more easier to obtain, the existing CXR datasets are much larger than the CT datasets for COVID-19 diagnosis. However, according to some prior studies [102], when X-ray images were taken from people affected by the COVID-19, some inconsistencies were observed in those X-ray Images. In addition, the CXR images have a high spatial resolution, but they are planer images and all structures visualised at CXR are displayed on a single plane, thus, they do not allow three-dimensional slicing [103].

\subsection{COVID-19 Detection through Mobile Sensors (DMS)}

To help in tracking different categories of illness and diseases, such as mental, cardiovascular, respiratory, and neurological, smartphone technology is found to be promising convenient biosensor [104], [105], [106], [107], [108] and for pandemic control, smartphone apps are a valuable commodity that could be tailored as respiratory monitoring systems [109]. Moreover there are some studies [105], [110], [111], [112] that and have analyzed a variety of acoustic feature types and accuracy of algorithms based on machine learning by utilizing audio recordings collected via smartphones for the automatic detection of respiratory illness.

In this pandemic of COVID-19, remote smartphone digital health technology has several advantages over traditional clinical visit for the screening and diagnosing COVID19. If COVID-19 infections are detected in early stages the rate of its transmission can be reduced by alerting the individuals to take precautions more actively [113]. Firstly. by using individual's everyday smartphone devices at home, workplace or vehicle, health screening implementation can be conducted on a large scale via an app. and respiratory health assessments can be performed remotely that can assist to quickly log the location/time of symptoms or incidence in real time. Secondly, overburdening at healthcare emergency clinics can be reduced by remote evaluations and cross infection at medical clinics and healthcare-related expenses can be minimized while serving. Thirdly, screening access can be provided to those individuals who live in remote place $s$ or where medical experts might be limited or not available.

\subsection{COVID-19 Detection through Sound Analysis}

To assist in the early diagnosis and detection of various illness, there are several opportunities in using human speech and other audio modalities and human generated audio is used as biomarkers by the researchers, thus, offering enormous benefits in the form of affordable solutions. For example, in Parkinson's disease, due to the lack of coordination of vocal muscles, the speech softness is prominent in patient's voice/speech [114], [115]. In coronary artery disease, due to the hardening of the arteries, the patients voice production (voice frequency) is affected [116]. For the detection of invisible diseases like traumatic brain injury, post-traumatic stress disorder and psychiatric conditions, some speech related features are also correlated including pitch, vocal tone, rate, rhythm and volume. Moreover, the researchers have explored glottal speech features such as glottal-to-noise excitation for the measurement of differences in excitation cycle, vocal tract airflow and fundamental frequency for the detection of respiratory diseases [112] and Suprasegmental prosodic-based speech features, such as loudness, pitch, and formant frequencies for the detection of COPD/asthma-related illness [111].

To date, the most of the common symptoms of COVID19 patients include dry cough, fever, muscle pain, fatigue, 
headache, diarrhea, sore throat, conjunctivitis and loss of taste and smell. when the condition is more severe, COVID19 patients feel severe chest pain, shortness of breadth and loss of speech or movement [117]. However, respiratory and non-respiratory diseases such as tuberculosis, bronchitis, asthma, gastroesophageal and reflux disease etc have the same symptoms as COVID-19 [118]. Due to this symptomology overlap, it is very challenging to develop new methods that can specifically identify and distinguish between COVID-19 and other respiratory diseases. Recently, researchers have explored the difference between the respiratory sounds of COVID-19 patients and healthy people [119], [120].

Since, COVID-19 virus critically affects the human speech production system and most of the symptoms of COVID-19 are associated with the functioning of the respiratory system, COVID-19 detection can be performed by analyzing three types of human generated audio or sound signals; speech signals, cough signals and breathing signals. A characteristic symptom of COVID-19 is the dry cough in which no mucous or phlegm is produced with the cough. To help in the current scenario of COVID-19, in various studies [34], [35], [121], the authors have discussed possible opportunities, solutions and use cases by leveraging human speech analysis. When respiratory muscles contract, the acoustic sound of a cough is generated. Cough (which can be wet, dry, or a wheezing, and whooping cough) is a sudden air expulsion from the nasal airways or throat which is characterized by a distinctive sound [122]. A typical cough sound signal consists of three phases; a rapid explosive phase, an intermediate decaying phase, a voiced phase and dry cough is characterized by all these three phases due to the absence of any mucus or sputum.

Cough is a predominant symptom of COVID-19 and thus, for possible preliminary screening and diagnosing COVID-19, researchers have utilized human cough by identifying COVID-19 specific cough and differentiating it from other similar sounds such as speech and laughter. They are motivated to analyze human cough for COVID-19 detection because prior studies [123], [124], [125], [126] have shown that cough from distinct respiratory syndromes have distinct latent features which are extracted by applying appropriate signal processing and mathematical transformations to train AI or Deep learning models for diagnostic purposes. In understanding the impact of COVID-19 on healthy speech production and the changes in voice of COVID19 infected patients, several initiatives have been taken as recently in a study [127], it has shown that approximately 25-32\% COVID-19 patients form the sampled populations in the UK and USA have a hoarse glottal voice quality [128]. Another study was conducted on voice pathology which was an indication of abnormally high rates of vocal dysphonia in COVID-19-positive individuals with mild to moderate severity because of the inflammation in tissues and glottic edema (vocal folds). [129].

\section{Challenges in combating COVID-19}

In fighting against COVID-19 pandemic, massive research proposals have been flooded regarding various aspects of COVID-19 detection but still there are unresolved challenges and research questions in COVID-19 detection that need to be addressed through developing novel solutions. In this section, we devise a taxonomy for these challenges and categorize them according to COVID-19 Data, Diagnosis and Regulations.

\subsection{Challenges concerning COVID-19 data}

In this subsection, we present two potential challenges related to COVID-19 data that the researchers are facing in its detection. These challenges are Security and privacy of COVID-19 patient's data and lack of sufficient standards of COVID-19 datasets.

\subsubsection{Security and privacy of patient's data}

To control the situation in this pandemic of COVID-19, and to make immediate actions and decisions and make up-todate policies in the face of public health issues, the authorities and governments aim to collect a range of personal information such as ID, contact number, personal medical data, diagnosis reports, CT scans and X-ray images, travel trajectory, and daily activities and share it with public health departments. This collection and sharing of highly sensitive patient data have raised concerns regarding his/her mobility, medical, and social data. It has also generated numerous privacy and security issues due to the open source nature of data and mandatory usage of advisory and contact tracing applications. This is because such applications collect sensitive personal health data and do not give clear and transparent privacy notices while briefing about the functions of the application, integrating customs and travel records with national healthcare database, using applications that via Bluetooth, collect the records of other people who have been in their close proximity within the past 21 days and insecure disposal and erasure of data and monitoring the location of individuals who are required to undergo home quarantine via cellular signals from their mobile phones.

Although, usage of tracing apps and digital technologies for monitoring and curtailing the virus spread rates seems to be promising especially in the time of lockdown but, these tools can raise privacy and security concerns just like many emerging technological advancements. Thus, anonymization of individual's information and ensuring the privacy preservation of COVID-19 patients personal and medical data is a challenging task [130], [131]. Another major concern is that workforce shift from office based to more remote work arrangements on permanent basis, hackers are exploiting this disruption in normal work patterns to hide intrusion activities and as a result, there is an increase in false positives in intrusion/risk alerts and complexity in filtering the false positives from actual positives. Due to successful cyberattacks, hospital operations are negatively affected, access to clinical services is delayed, leading to significant economic loss [132], [133]. In addition, sharing COVID-19 patients data for academic purposes has also resulted into public hatred and discrimination as reported in [134]. Therefore, this situation demands complete anonymity of medical and mobility data and there is a need to carefully manage these concerns by implying security controls such as encryption and anonymization to deter data leaks and manipulation attempts form non trusted third 


\begin{tabular}{|c|c|}
\hline Challenges & Research Questions \\
\hline $\begin{array}{l}\text { Security and privacy of patient's } \\
\text { data }\end{array}$ & $\begin{array}{l}\text { - How to make the collection of COVID-19 Patient's data a transparent process by informing } \\
\text { individuals with whom it will be shared? } \\
\text { - How to securely store patient's sensitive medical and personal data with limited access controls? } \\
\text { - How sensitive medical data of COVID-19 patients can be anonymized, aggregated, and encrypted? } \\
\text { - How to develop security and encryption systems for the protection of patient privacy and to ensure } \\
\text { data security by making the patients aware of privacy choices so that disclosure of sensitive personal } \\
\text { information can be avoided? }\end{array}$ \\
\hline $\begin{array}{l}\text { Lack of sufficient standard COVID- } \\
19 \text { datasets }\end{array}$ & $\begin{array}{l}\text { - How to develop robust and statistically stable deep learning models which can learn bias? } \\
\text { - How the models can make predictions confidently for an unseen biased data set with similar values } \\
\text { of performance metrics? } \\
\text { - How to designing a fair testing protocol which can avoid misleading results when different datasets } \\
\text { are merged with large differences among them? }\end{array}$ \\
\hline $\begin{array}{l}\text { Detecting and Diagnosing without } \\
\text { or mild symptoms }\end{array}$ & $\begin{array}{l}\text { - How to capture noiseless breathing patterns or signals through smartphone sensors while the } \\
\text { patients is wearing a facial mask? } \\
\text { - How to develop deep learning models that are robust against multicultural or local and multilingual } \\
\text { speech and sound variability? }\end{array}$ \\
\hline $\begin{array}{l}\text { User acceptance of remote health- } \\
\text { care and Tele-health/Telemedicine }\end{array}$ & $\begin{array}{l}\text { - How to learn to integrate tele-medicine as one option in our routine care in a more efficient way? } \\
\text { - How to implement care pathways for different patient populations while determining the right } \\
\text { patient phenotype and dose of telemedicine for optimizing convenience and efficiency? } \\
\text { What are the ways to implement telehealthcare that is financially sustainable and maximizes patient } \\
\text { - } \quad \text { Howety and privacy? } \\
\text { - How to develop patients trust upon digital systems and telehelath services applications? } \\
\text { requirements of professionals, health-care organisations and regulatory? }\end{array}$ \\
\hline $\begin{array}{l}\text { Policies and Regulations for Detect- } \\
\text { ing COVID-19 }\end{array}$ & $\begin{array}{l}\text { - What are the policies for data sharing in telehealth systems? } \\
\text { - How to make regulations, policies and standards while considering different cultures and forming a } \\
\text { balance between interests of individuals and that of population? } \\
\text { What are steps that governments of each country has to take for minimizing the impact of fake news } \\
\text { during COVID, within its own political system? } \\
\text { Which polices and strategies might be effective in controlling the outbreak in this COVID-19 } \\
\text { pandemic with indifferent attitude of the public? }\end{array}$ \\
\hline $\begin{array}{l}\text { Misconeptions, Misinformation and } \\
\text { Misdiagnosis in COVID-19 epidemic }\end{array}$ & $\begin{array}{l}\text { - How to develop the deep learning models and techniques that can detect and identify COVID-19 } \\
\text { fake news or rumors form true information about COVID-19 spread more accurately while utilizing } \\
\text { a small and multilingual dataset? } \\
\text { - How to identify and investigate the origin behind misinformation and disinformation shring from } \\
\text { social media posts and how to overcome fake health information regrading COVID-19 by using } \\
\text { interactions based on health evidence with social impacts? } \\
\text { - How the mood of the population with different demography (e.g age,gender) can be handled } \\
\text { while containing the public concern at desirable levels and utilizing this knowledge to combat } \\
\text { misinformation propagation? } \\
\text { - How to model the reaction of people with different demographics to misinformation about COVID- } \\
19 \text { spread and the information presented to them in the form of alerts and warnings by keeping a } \\
\text { smooth balance between raising attention and providing assurance? }\end{array}$ \\
\hline
\end{tabular}

parties from as well as to assure that the results of COVID19 detection are optimum. Moreover, during the design and refresh stages of contact tracing applications, privacy principles such as "privacy by design" and "privacy by default" should be considered. Although some efforts have been made by leveraging technologies such as blockchain [135], [136], [137], federated learning [138], [139], [140], [141] and incentive based mechanisms [142].

\subsubsection{Lack of sufficient standard COVID-19 datasets}

Data is a key component in machine learning and without sufficient data, for solving a problem, deep learning approaches may experience a limitation in their effectiveness, accuracy and efficiency. Thus, in the current pandemic, lack of standard and adequate COVID-19 clinical data prompted as a barrier and a severe challenge in the research of COVID19 detection and diagnosis, limiting the performance of deep learning based COVID-19 diagnostic and prognosis tools and techniques [143]. Due to the rapid explosion of COVID19 , and urgency, scientific datasets were often constructed in a quick manner and thus the collected datasets are small and inadequate and may not be as precise as they should be. In this regard an effort has been made in [144], where GAN is used to generate more X-ray images and develop a COVID-19 diagnostic tool. With smaller datasets, researchers have exploited pre-trained deep learning algorithms which usually worked well on larger datasets but here on smaller datasets, their performance has diminished due to overfitting problems [145].

It is thus, challenging to develop deep learning approaches for dealing with smaller as well as imbalanced datasets. Moreover, COVID-19 data is collected using smartphones or other voice recorders in unconstrained environments which are generally noisy and contain reverberation. Hence, the available imaging data for COVID-19 patients are in bad quality, noisy, incomplete, and in some cases the 
labels are inaccurate and ambiguous [36]. It is complex to train a deep learning algorithm on such data while resolving data redundancy, sparsity and also the missing values. Consequently, insufficient time series data and low quality data result in biased and inaccurate predictions and unreliable results [53]. It is thus, challenging for deep learning algorithms to diagnose and detect COVID-19 accurately and efficiently. In addition, because of using different datasets with different numbers of samples, definitively concluding which system yields the best result for COVID-19 detection is quite difficult. There is a need to establish organized framework and datasets, to make them easily accessible for the researchers around the world.

\subsection{Challenges concerning Smartphone based COVID- 19 detection/diagnosis}

In this subsection, we present two significant challenges related to COVID-19 detection/diagnosis through smartphones that the researchers are currently facing for its efficient detection. These challenges are Detecting and Diagnosing COVID-19 patients with no or mild symptoms and User acceptance of remote healthcare and Telehealth/Telemedicine.

\subsubsection{Detecting and Diagnosing COVID-19 patients with no or mild COVID-19 symptoms}

Currently, to control the world-wide disruptive and spreading novel coronavirus disease, smartphones, with their computing proficiency, have been used to detect COVID-19 by collecting and analyzing speech, cough and breathing signals. Some interesting frameworks based on mobile smartphones are provided for developing COVID-19 detection soft wares and algorithms [61], [69], [146], [147]. Moreover, smartphones are also capable of processing X-ray images and CT scans by using deep learning in smartphones for the detection of COVID-19 [148]. Deep learning algorithms work well by providing better insights and accuracy in diagnosis with larger datasets but using them on smartphones may degrade their performance because the computing capability of a mobile to treat a large amount of data is lower than a grand machine or a computer and thus, to accomplish this task, lightweight algorithms are required to develop which is a challenging task. There are also some other noticeable challenges in this regard. Experiments performed in [149] have shown that the quality of images in this way is not adequate for managing the smartphone based applications. It is also challenging to get accurate and relevant speech data for developing deep learning models in terms of social distancing norms. To assist in this regard, chat bots play a significant role and automate the screening of COVID-19 making it faster but it is also a challenging task to design chat bots while considering its positive and negative impacts.

In a recent pathology study [129] there was an indication of abnormally high rates of vocal dysphonia in COVID19 patients with mild to moderate severity because of glottic edema and tissue inflammation. A major problem is that most of the smartphone based studies [109] have not scrutinized speech data but they have only examined recorded breathing/cough sounds for COVID-19 detection the individuals with moderate COVID-19-like symptoms that tested negative are ignored. Thus, these studies are not effective in cases when individuals do not have difficulties in breathing or cough symptoms. It is therefore challenging to develop smartphone based systems that can detect COVID-19 patients with no or mild symptoms such as cough.

\subsubsection{User acceptance of remote healthcare and Tele- health/Telemedicine}

Information and communication technologies are playing a significant role in assisting doctors, practitioners and healthcare professionals and workers for faster diagnosis of viral infections and diseases. In the COVID-19 epidemic due to the need for social distancing and in-person contact restrictions, telehealth services application and remote care or remote diagnosis has accelerated and are used in the large scale screening of patients, superviisng patient care by experts and for remote clinical encounters [150], [151]. It is made possible with the help of body wearable sensors, tele-healthcare, telemedicine and AI-chatbots [152], [153]. By using telehealth, healthcare professionals are able to provide medical services at a distance using video imaging and videoconferencing and other technologies. When emergency situations are detected, the smart homes equipped with environmental and personal sensors (interconnected using the IoT) are capable of monitoring patient health and sending messages to responsible clinicians. Also, health Wearable devices have a myraid of different sensors that can collect distinct data types such a s steps, sleep, heart rate pulse.

By processing the collected sensor data and applying deep learning and statistical methods, wearable devices are found to be promising technology to track viral infections overtime as well for detecting COVID-19 and proactively detect them before their onset. However, challenges are raised because environmental conditions and external factors affect the physiological measurements resulting into inherently unpredictable time-series data. This unpredictability in the data leads to low accuracy of models and systems which in turn badly shatter the patient's trust upon these systems. When patients and providers are unsatisfied with Telemedicine, rather to be subjected a bad experience, they both may prefer to suffer the inconvenience and unavailability of in-person care. Moreover, mostly patients also prefer to see their own health care provider as opposed to someone with whom they have no established relationship. Thus, patients trust upon telehealth should be developed which is a challenging task [154]. Due to the dramatic improvements in technology, digital availability has increased accessibility and quality of care and at the time of epidemic conditions such as COVID-19, telemedicine has the potential to control the disease and management of clinical case and to improve research of epidemiological [155], [156], [157]. The patient's general appearance including patient's respirations (effort of breathing, speech,accessory respiratory muscle involvement), oropharynx observation, patientdirected lymph nodes to assess for notable lymphadenopathy, presence of a patient cough (dry or wet) can be noted via video [158]. But despite of this, the concept of telehealth and telenursing is not a panacea, coming up with comes 


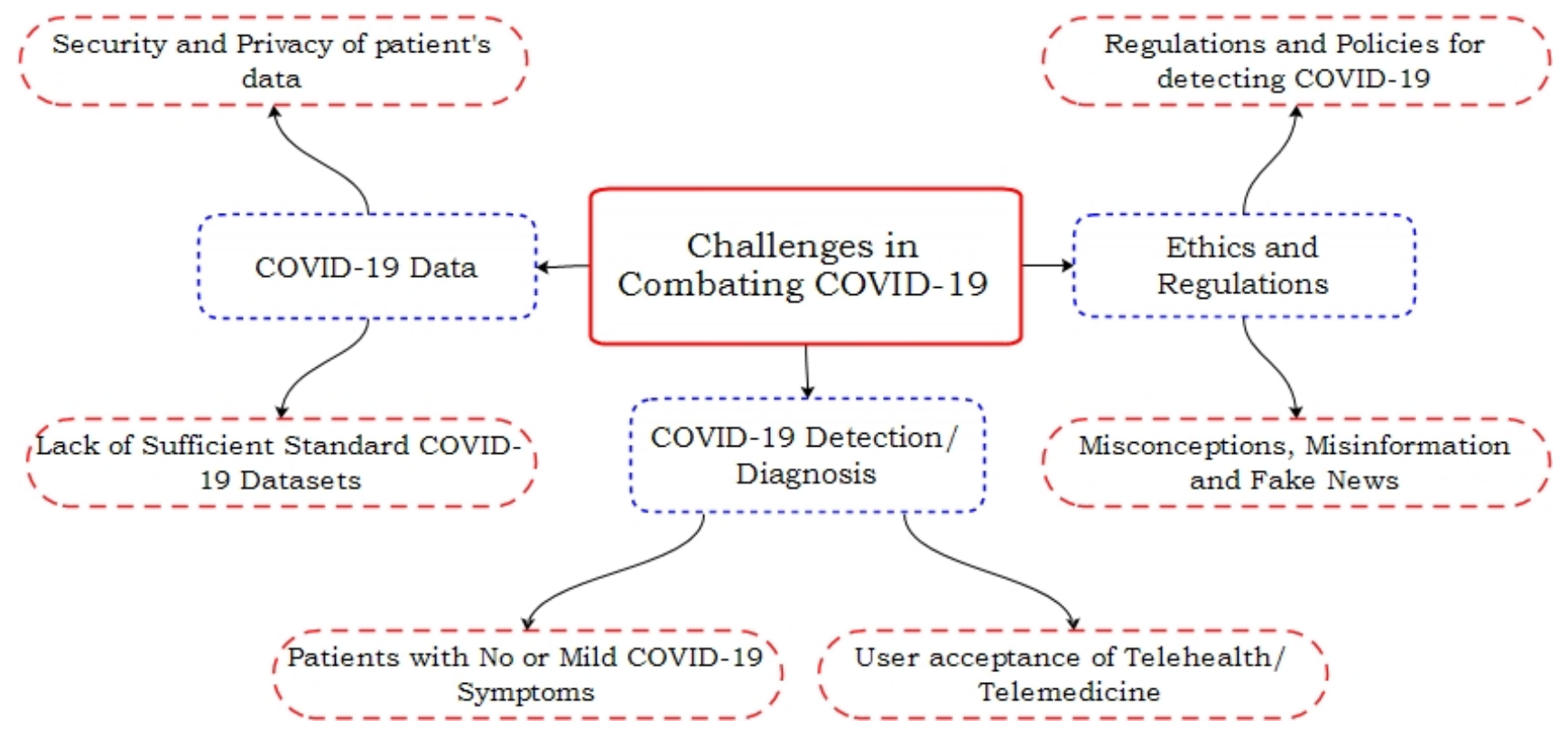

Fig. 4. Challenges in Combating COVID-19 Pandemic

with risks including security breaches and hindrances in its wide adoption still exist [159].

The most important hinderance in wide adoption of telemedicine is the patients preference of Face-to-Face Healthcare. A major problem is that there are some health issues such as transplantation, therapies etc that can only be handled and evaluated in person and using telemedicine it may have trouble concentrating or working remotely. Also, hurdles in implementing telehealth care programs also largely depend on payments systems, insurance and accreditation as there is a lack of supportive payment structures and heavy regulatory laws due to which telemedicine has yet to be widely implemented [151], [160]. Some other noticeable barriers that currently exist to telemedicine include a lack of knowledge about having telemedicine visits as an optiona, lack of education about the efficacy and safety of telemedicine in light of current circumstances and a lack of understanding about how to access telemedicine visits [161]. Although some patients are already embracing telehealth mode of care delivery [162], [163], however, healthcare professionals need to be aware of patients preferences for communication and to learn new skills for conducting telemedicine visits by developing programs to educate and mentor colleagues and trainees [164] and find the ways of using telehealth by which the need of patients can be met with the levels of attentiveness, expertise and empathy that is provided during office clinical visits.

\subsection{Challenges concerning Ethics and Regulations}

In this subsection, we present two critical challenges related to ethics and regulations in detecting COVID-19 that the research community is facing currently. These challenges are the lack of regulations and policies for detecting COVID19 and controlling misconceptions, misinformation and fake news about the pandemic.

\subsubsection{Lack of Regulations and Policies for detecting COVID-19}

The daily number of confirmed cases (infected and dead) is considerably increasing. To control this outbreak, governmental authorities are defining different policies and are playing a vital role by applying various approaches such as screening and testing at a large scale, lockdown, social distancing etc. For example, Korean government has started the quarantine policy from 1st April 2020 according to which it is compulsory to quarantine all the passengers for 14 days at designated facilities or registered addresses who are are entering Korea. Moreover, all the passengers are obliged to send their daily self-diagnosis reports taken through selfdiagnosis apps installed in their mobile phones. In case if people don't have mobile phones and cannot install the self-diagnosis apps, the Seoul Metropolitan Government has implemented an AI monitoring calling system which can automatically check the health conditions of the people [165]. It is still challenging to make effective policies and regulations as individual citizens and businesses usually ignore what is asked of them.

There is a need for rapid development of new policies and additional restrictive measures, to meet the novel circumstances. FFor pandemics, the rationing policies vary from country to country on the basis of their health system, institution and jurisprudence and thus the COVID-19 pandemic has also raised a host of ethical challenges, due to rationing of scarce critical care resources by the health care systems. Some countries have uniform policy while some have no policies at all. Thus, there is need to ensure that remote healthcare practices are carried out in an ethical, confidential, and safe manner by establishing laws and regulations around the world. Moreover, to preserve many of the current waivers that permit telemeidicine services and to make telemedicine a viable solution, there is a need to advocate for legislative reform at the federal and state levels. From an ethical perspective, to avoid overt and implicit bias, 
there is a dire need to apply triage policies consistently to all patients. From a legal perspective, allocation decisions and triage policies should not be unlawful against protected classes of individuals (e.g on the basis of race, religion or color, national origin, sex ) so that discrimination can be avoided [166].

\subsubsection{Controlling Misconceptions, Misinformation and fake news in the COVID-19 epidemic}

In COVID-19 pandemic across the globe, Medicine and Science is combating against it with the assistance of digital technologies, but the healthcare systems are in disarray and they are facing another critical problem, fake news or misinformation which refer to fabrications, satires, and hoaxes mixed in real reports to mislead people's judgments, especially during the pandemic. It is also stated by World Health Organization that pandemic is accompanied by an "infodemic" (information epidemic). Fake news is intentionally written to mislead readers to believe false information, which makes it difficult and nontrivial to detect based on news content. Fake news is divided into two categories; misinformation and disinformation. Misinformation refers to the false or misleading information that is inadvertently shared and disinformation refers to deliberate creation and sharing of false information that is purposely spread to deceive people and is known to be false [167], [168].

With the start of COVID-19 pandemic, the spreading of inaccurate information online., i.e, a cluster of fake news about possible remedies, vaccinations and lockdown policies, are not only life threatening but also causing disruptions in society, creating confusion among people and even leading to deadly consequences in health problems. In addition, the prevalent misinformation is also disrupting the social order and the supply chain disrupted out of fear since people started to pile up stocks of masks and sanitizers [169]. The misinformation in the form of numerous harmful advice or cures being suggested regarding the prevention and treatment of COVID-19 is taken seriously which has caused the deterioration of the patient's condition as well as the emergence of new diseases [170]. For instance it is suggested that drinking fish tank additives, bleach, or cow urine are suggested to be a cure for COVID-19 which are actually harmful for health [171]. Particularly, one major concern is the misinformation regarding COVID-19 vaccines which is the main cause of vaccine hesitancy and is fueled by rumors of safety and conspiracies [172], thus, influencing people's willingness to follow the recommendations by health and political authorities on vaccination [173]. Due to these rumors people either refuse to vaccinate or delay its acceptance despite the availability of vaccination services [174]. This is mainly because the online social media lack gate keeping and proper regulations and thus, it is a place to disseminate misinformation and fake news rapidly [175] by providing a fertile ground for spreading a large amount of unfiltered content [176] and authorizing a misinformation phenomenon. Moreover, to misguiding news are presented purposefully by many online news portals in order to increase their popularity. This pandemic is seen as a good opportunity for the journalists, bloggers or anyone with access to social media to increase the number of their followers or readership rates and to obtain extra attention by publishing
COVID-19 related information. in doing this process, there is manipulation or fabrication of valid information which create fake news or stories in order for it to appear more interesting to the public [177]. Consequently, the public's perception of reality is possibly manipulated and aggravated through the dissemination of fake news content [178].

During this epidemic, these fake news and rumors are creating panic among the masses and thus it is very crucial to detect [179] Fake news. In most of the existing methods for fake news detection, false news is identified by combining richer data, which provide a data repository including news contents, social contents as well as dynamic information. However, such methods cannot be applied in new fields due to the lack of universality. Hence, researchers have proposed semi-supervised and unsupervised methods [180], [181], [182], [183], which try to make a trade-off between the amount of training data and the final training accuracy. It is still a challenging task to detect COVID19 fake news as the COVID-19 fake news is multilingual. Moreover, the accuracy of some of the above mentioned recent systems have not yet reached acceptable levels because of the insufficient learning of limited corpus contents and the incomplete hard samples mining. The size and features of data used by the researchers is not enough for building models for accurate classification. Moreover, for accurate classification of fake news detection, very few research has been done towards application of linguistic and deep learning techniques. In addition, the model's understanding of common sense is diminished due to the excessive further-training, resulting in some incomprehensible mistakes. There is a need to design an effective detector which can efficiently and accurately differentiate between the real/actual news and fake news according to its title or summary. For evaluating the proposed works in the current pandemic, there is a need to apply the existing NLP techniques for fake news identification on COVID19 social media data-sets [184]. From ethical point of view, the authorities and people should be aware of their ethical duties so as to ensure that only ethical and valid information is being shred on social media [177].

\section{CONCLUSION}

Severe acute respiratory syndrome coronavirus 2 (SARSCoV-2) disease (COVID-19) has profound effect on the well being worldwide and has significantly impacted global health and socio-economics systems. It has become a major global concern because it is spreading at a fast rate and the number of diseases related deaths continues to grow globally. To find a viable cure against the COVID-19, medical and scientific researchers are working rapidly and restlessly with the aid of artificial intelligence (AI) and deep learning (DL) in numerous ways. In contribution to fight against COVID-19, DL algorithms more suitably CNN and its variants have been successfully applied for screening, diagnosis, prediction, severity checking, drug discovery and treatment of COVID-19. This survey offers the identification of significant challenges in deep learning based COVID19 detection. In this survey, we have first presented and explained general pipeline of image processing with regard to DL based COVID-19 detection. Then we have presented 
the taxonomy of modalities used for DL based COVID-19 detection methods and explained each category. Moreover, we have also identified and presented potential challenges and devised a taxonomy for the challenges in DL based detection of COVID-19. Important research questions for each category of challenges are also identified. This survey opens a promising path and a reference guide for the researchers contributing in the battle against COVID-19.

\section{References}

[1] M. E. El Zowalaty and J. D. Järhult, "From sars to covid-19: A previously unknown sars-related coronavirus (sars-cov-2) of pandemic potential infecting humans-call for a one health approach," One Health, vol. 9, p. 100124, 2020.

[2] M. A. Soghaier, K. M. Saeed, and K. K. Zaman, "Public health emergency of international concern (pheic) has declared twice in 2014; polio and ebola at the top," AIMS public health, vol. 2, no. 2, p. 218, 2015.

[3] C. Huang, Y. Wang, X. Li, L. Ren, J. Zhao, Y. Hu, L. Zhang, G. Fan, J. Xu, X. Gu et al., "Clinical features of patients infected with 2019 novel coronavirus in wuhan, china," The lancet, vol. 395, no. 10223, pp. 497-506, 2020.

[4] E. C. for Disease Prevention and C. (ECDC), "Geographical distribution of 2019-ncov cases," 2020.

[5] S. A. Tarasov, V. V. Zarubaev, E. A. Gorbunov, S. A. Sergeeva, and O. I. Epstein, "Retracted: Activity of ultra-low doses of antibodies to gamma-interferon against lethal influenza a (h1n1) 2009 virus infection in mice," 2012.

[6] A. M. Zaki, S. Van Boheemen, T. M. Bestebroer, A. D. Osterhaus, and R. A. Fouchier, "Isolation of a novel coronavirus from a man with pneumonia in saudi arabia," New England Journal of Medicine, vol. 367, no. 19, pp. 1814-1820, 2012.

[7] R. Keni, A. Alexander, P. G. Nayak, J. Mudgal, and K. Nandakumar, "Covid-19: emergence, spread, possible treatments, and global burden," Frontiers in public health, vol. 8, p. 216, 2020.

[8] P. Zhou, X.-L. Yang, X.-G. Wang, B. Hu, L. Zhang, W. Zhang, H.R. Si, Y. Zhu, B. Li, C.-L. Huang et al., "A pneumonia outbreak associated with a new coronavirus of probable bat origin," nature, vol. 579, no. 7798, pp. 270-273, 2020.

[9] Y.-R. Guo, Q.-D. Cao, Z.-S. Hong, Y.-Y. Tan, S.-D. Chen, H.-J. Jin, K.-S. Tan, D.-Y. Wang, and Y. Yan, "The origin, transmission and clinical therapies on coronavirus disease 2019 (covid-19) outbreak-an update on the status," Military Medical Research, vol. 7, no. 1, pp. 1-10, 2020.

[10] K. G. Andersen, A. Rambaut, W. I. Lipkin, E. C. Holmes, and R. F. Garry, "The proximal origin of sars-cov-2," Nature medicine, vol. 26, no. 4, pp. 450-452, 2020.

[11] S. Bhattacharya, P. K. R. Maddikunta, Q.-V. Pham, T. R. Gadekallu, C. L. Chowdhary, M. Alazab, M. J. Piran et al., "Deep learning and medical image processing for coronavirus (covid19) pandemic: A survey," Sustainable cities and society, vol. 65, p. 102589, 2021.

[12] G. Kampf, D. Todt, S. Pfaender, and E. Steinmann, "Persistence of coronaviruses on inanimate surfaces and their inactivation with biocidal agents," Journal of hospital infection, vol. 104, no. 3, pp. 246-251, 2020

[13] M. A. Shereen, S. Khan, A. Kazmi, N. Bashir, and R. Siddique, "Covid-19 infection: Origin, transmission, and characteristics of human coronaviruses," Journal of advanced research, vol. 24, pp. 91-98, 2020.

[14] A. Afzal, "Molecular diagnostic technologies for covid-19: Limitations and challenges," Journal of advanced research, 2020.

[15] L. Wang, Z. Q. Lin, and A. Wong, "Covid-net: A tailored deep convolutional neural network design for detection of covid-19 cases from chest x-ray images," Scientific Reports, vol. 10, no. 1, pp. 1-12, 2020.

[16] J. Kanne, B. Little, J. Chung, B. Elicker, and L. Ketai, “Essentials for radiologists on covid-19: An update-radiology scientific expert panel." Radiology, pp. 200527-200 527, 2020.

[17] B. Sahiner, A. Pezeshk, L. M. Hadjiiski, X. Wang, K. Drukker, K. H. Cha, R. M. Summers, and M. L. Giger, "Deep learning in medical imaging and radiation therapy," Medical physics, vol. 46, no. 1, pp. e1-e36, 2019.
[18] F. Piccialli, V. Di Somma, F. Giampaolo, S. Cuomo, and G. Fortino, "A survey on deep learning in medicine: Why, how and when?" Information Fusion, vol. 66, pp. 111-137, 2021.

[19] P. Rajan Jeyaraj and E. R. S. Nadar, "Retracted: Atrial fibrillation classification using deep learning algorithm in internet of thingsbased smart healthcare system," Health informatics journal, vol. 26, no. 3, pp. 1827-1840, 2020.

[20] S. Tuli, N. Basumatary, S. S. Gill, M. Kahani, R. C. Arya, G. S. Wander, and R. Buyya, "Healthfog: An ensemble deep learning based smart healthcare system for automatic diagnosis of heart diseases in integrated iot and fog computing environments," Future Generation Computer Systems, vol. 104, pp. 187-200, 2020.

[21] M. Liu, M. Zhou, T. Zhang, and N. Xiong, "Semi-supervised learning quantization algorithm with deep features for motor imagery eeg recognition in smart healthcare application," Applied Soft Computing, vol. 89, p. 106071, 2020.

[22] K. Muhammad, S. Khan, J. Del Ser, and V. H. C. de Albuquerque, "Deep learning for multigrade brain tumor classification in smart healthcare systems: A prospective survey," IEEE Transactions on Neural Networks and Learning Systems, 2020.

[23] I. I. Baskin, "The power of deep learning to ligand-based novel drug discovery," Expert opinion on drug discovery, vol. 15, no. 7, pp. 755-764, 2020.

[24] F. Gentile, V. Agrawal, M. Hsing, A.-T. Ton, F. Ban, U. Norinder, M. E. Gleave, and A. Cherkasov, "Deep docking: a deep learning platform for augmentation of structure based drug discovery," ACS central science, vol. 6, no. 6, pp. 939-949, 2020.

[25] S. Korkmaz, "Deep learning-based imbalanced data classification for drug discovery," Journal of Chemical Information and Modeling, vol. 60, no. 9, pp. 4180-4190, 2020.

[26] X. Ma, Y. Niu, L. Gu, Y. Wang, Y. Zhao, J. Bailey, and F. Lu, “Understanding adversarial attacks on deep learning based medical image analysis systems," Pattern Recognition, vol. 110, p. 107332, 2021.

[27] S. Budd, E. C. Robinson, and B. Kainz, "A survey on active learning and human-in-the-loop deep learning for medical image analysis," Medical Image Analysis, p. 102062, 2021.

[28] G. Verma and S. Prakash, "Pneumonia classification using deep learning in healthcare," International Journal of Innovative Technology and Exploring Engineering (IJITEE), vol. 9, no. 4, pp. 1715-1723, 2020.

[29] X. Xu, X. Jiang, C. Ma, P. Du, X. Li, S. Lv, L. Yu, Q. Ni, Y. Chen, J. Su et al., "A deep learning system to screen novel coronavirus disease 2019 pneumonia," Engineering, vol. 6, no. 10, pp. 11221129, 2020.

[30] F. Shi, J. Wang, J. Shi, Z. Wu, Q. Wang, Z. Tang, K. He, Y. Shi, and D. Shen, "Review of artificial intelligence techniques in imaging data acquisition, segmentation and diagnosis for covid-19," IEEE reviews in biomedical engineering, 2020.

[31] W. Hariri and A. Narin, "Deep neural networks for covid-19 detection and diagnosis using images and acoustic-based techniques: A recent review," arXiv preprint arXiv:2012.07655, 2020.

[32] N. Benameur, R. Mahmoudi, S. Zaid, Y. Arous, B. Hmida, and M. H. Bedoui, "Sars-cov-2 diagnosis using medical imaging techniques and artificial intelligence: A review," Clinical Imaging, 2021.

[33] A. Bhargava and A. Bansal, "Novel coronavirus (covid-19) diagnosis using computer vision and artificial intelligence techniques: a review," Multimedia Tools and Applications, pp. 1-16, 2021.

[34] G. Deshpande and B. Schuller, "An overview on audio, signal, speech, \& language processing for covid-19," arXiv preprint arXiv:2005.08579, 2020.

[35] G. Deshpande and B. W. Schuller, "Audio, speech, language, \& signal processing for covid-19: A comprehensive overview," arXiv preprint arXiv:2011.14445, 2020.

[36] M. M. Islam, F. Karray, R. Alhajj, and J. Zeng, "A review on deep learning techniques for the diagnosis of novel coronavirus (covid-19)," IEEE Access, vol. 9, pp. 30551-30 572, 2021.

[37] H. Chiroma, A. E. Ezugwu, F. Jauro, M. A. Al-Garadi, I. N. Abdullahi, and L. Shuib, "Early survey with bibliometric analysis on machine learning approaches in controlling covid-19 outbreaks," PeerJ Computer Science, vol. 6, p. e313, 2020.

[38] S. Rajpal, N. Lakhyani, A. K. Singh, R. Kohli, and N. Kumar, "Using handpicked features in conjunction with resnet-50 for improved detection of covid-19 from chest x-ray images," Chaos, Solitons \& Fractals, vol. 145, p. 110749, 2021. 
[39] U. S. SM, R. Ganesan, J. Katiravan, M. Ramakrishnan et al. "Mobile application based speech and voice analysis for covid19 detection using computational audit techniques," International Journal of Pervasive Computing and Communications, 2020.

[40] A. Bernheim, X. Mei, M. Huang, Y. Yang, Z. A. Fayad, N. Zhang, K. Diao, B. Lin, X. Zhu, K. Li et al., "Chest ct findings in coronavirus disease-19 (covid-19): relationship to duration of infection," Radiology, p. 200463, 2020.

[41] M. Abdel-Basset, V. Chang, H. Hawash, R. K. Chakrabortty, and M. Ryan, "Fss-2019-ncov: A deep learning architecture for semi-supervised few-shot segmentation of covid-19 infection," Knowledge-Based Systems, vol. 212, p. 106647, 2021.

[42] Y. Qiu, Y. Liu, S. Li, and J. Xu, "Miniseg: An extremely minimum network for efficient covid-19 segmentation," arXiv preprint arXiv:2004.09750, 2020.

[43] F. Shan, Y. Gao, J. Wang, W. Shi, N. Shi, M. Han, Z. Xue, D. Shen, and Y. Shi, "Abnormal lung quantification in chest ct images of covid-19 patients with deep learning and its application to severity prediction," Medical physics, vol. 48, no. 4, pp. 1633-1645, 2021.

[44] H.-t. Zhang, J.-s. Zhang, H.-h. Zhang, Y.-d. Nan, Y. Zhao, E.-q Fu, Y.-h. Xie, W. Liu, W.-p. Li, H.-j. Zhang et al., "Automated detection and quantification of covid-19 pneumonia: $\mathrm{Ct}$ imaging analysis by a deep learning-based software," European journal of nuclear medicine and molecular imaging, vol. 47, no. 11, pp. 25252532,2020

[45] B. Wang, S. Jin, Q. Yan, H. Xu, C. Luo, L. Wei, W. Zhao, X. Hou, W. Ma, Z. Xu et al., "Ai-assisted ct imaging analysis for covid-19 screening: Building and deploying a medical ai system," Applied Soft Computing, vol. 98, p. 106897, 2021.

[46] H. Yue, Q. Yu, C. Liu, Y. Huang, Z. Jiang, C. Shao, H. Zhang, B. Ma, Y. Wang, G. Xie et al., "Machine learning-based ct radiomics method for predicting hospital stay in patients with pneumonia associated with sars-cov-2 infection: a multicenter study," Annals of translational medicine, vol. 8, no. 14, 2020.

[47] K. He, W. Zhao, X. Xie, W. Ji, M. Liu, Z. Tang, Y. Shi, F. Shi, Y. Gao, J. Liu et al., "Synergistic learning of lung lobe segmentation and hierarchical multi-instance classification for automated severity assessment of covid-19 in ct images," Pattern Recognition, vol. 113, p. 107828, 2021.

[48] K. Gao, J. Su, Z. Jiang, L.-L. Zeng, Z. Feng, H. Shen, P. Rong, X. Xu, J. Qin, Y. Yang et al., "Dual-branch combination network $(\mathrm{dcn})$ : Towards accurate diagnosis and lesion segmentation of covid-19 using ct images," Medical image analysis, vol. 67, p. 101836, 2021.

[49] L. Hussain, T. Nguyen, H. Li, A. A. Abbasi, K. J. Lone, Z. Zhao, M. Zaib, A. Chen, and T. Q. Duong, "Machine-learning classification of texture features of portable chest x-ray accurately classifies covid-19 lung infection," BioMedical Engineering OnLine, vol. 19, no. 1, pp. 1-18, 2020.

[50] M. A. Elaziz, K. M. Hosny, A. Salah, M. M. Darwish, S. Lu, and A. T. Sahlol, "New machine learning method for image-based diagnosis of covid-19," Plos one, vol. 15, no. 6, p. e0235187, 2020.

[51] D. Gil, K. Díaz-Chito, C. Sánchez, and A. Hernández-Sabaté, "Early screening of sars-cov-2 by intelligent analysis of x-ray images," arXiv preprint arXiv:2005.13928, 2020.

[52] M. Tamal, M. Alshammari, M. Alabdullah, R. Hourani, H. A. Alola, and T. M. Hegazi, "An integrated framework with machine learning and radiomics for accurate and rapid early diagnosis of covid-19 from chest x-ray," medRxiv, 2020.

[53] E. Tartaglione, C. A. Barbano, C. Berzovini, M. Calandri, and M. Grangetto, "Unveiling covid-19 from chest x-ray with deep learning: a hurdles race with small data," International Journal of Environmental Research and Public Health, vol. 17, no. 18, p. 6933, 2020.

[54] Z. Wang, Y. Xiao, Y. Li, J. Zhang, F. Lu, M. Hou, and X. Liu, "Automatically discriminating and localizing covid-19 from community-acquired pneumonia on chest x-rays," Pattern recognition, vol. 110, p. 107613, 2021.

[55] M. M. Rahaman, C. Li, Y. Yao, F. Kulwa, M. A. Rahman, Q. Wang, S. Qi, F. Kong, X. Zhu, and X. Zhao, "Identification of covid19 samples from chest $\mathrm{x}$-ray images using deep learning: A comparison of transfer learning approaches," Journal of X-ray Science and Technology, no. Preprint, pp. 1-19, 2020.

[56] L. Li, L. Qin, Z. Xu, Y. Yin, X. Wang, B. Kong, J. Bai, Y. Lu, Z. Fang, Q. Song et al., "Using artificial intelligence to detect covid-19 and community-acquired pneumonia based on pulmonary ct: evaluation of the diagnostic accuracy," Radiology, vol. 296, no. 2, pp. E65-E71, 2020.

[57] S. H. Kassani, P. H. Kassasni, M. J. Wesolowski, K. A. Schneider, and R. Deters, "Automatic detection of coronavirus disease (covid-19) in x-ray and ct images: A machine learning-based approach," arXiv preprint arXiv:2004.10641, 2020.

[58] Y. Pathak, P. K. Shukla, A. Tiwari, S. Stalin, and S. Singh, "Deep transfer learning based classification model for covid-19 disease," Irbm, 2020.

[59] H. Kang, L. Xia, F. Yan, Z. Wan, F. Shi, H. Yuan, H. Jiang D. Wu, H. Sui, C. Zhang et al., "Diagnosis of coronavirus disease 2019 (covid-19) with structured latent multi-view representation learning," IEEE transactions on medical imaging, vol. 39, no. 8, pp. 2606-2614, 2020.

[60] F. Shi, L. Xia, F. Shan, B. Song, D. Wu, Y. Wei, H. Yuan, H. Jiang, Y. He, Y. Gao et al., "Large-scale screening to distinguish between covid-19 and community-acquired pneumonia using infection size-aware classification," Physics in Medicine $\mathcal{E}$ Biology, vol. 66, no. 6, p. 065031, 2021.

[61] C. Brown, J. Chauhan, A. Grammenos, J. Han, A. Hasthanasombat, D. Spathis, T. Xia, P. Cicuta, and C. Mascolo, "Exploring automatic diagnosis of covid-19 from crowdsourced respiratory sound data," in Proceedings of the 26th ACM SIGKDD International Conference on Knowledge Discovery \& Data Mining, pp. 3474-3484, 2020.

[62] P. K. Sethy, S. K. Behera, P. K. Ratha, and P. Biswas, "Detection of coronavirus disease (covid-19) based on deep features and support vector machine," 2020.

[63] I. D. Apostolopoulos and T. A. Mpesiana, "Covid-19: automatic detection from x-ray images utilizing transfer learning with convolutional neural networks," Physical and Engineering Sciences in Medicine, vol. 43, no. 2, pp. 635-640, 2020.

[64] R. Kumar, R. Arora, V. Bansal, V. J. Sahayasheela, H. Buckchash, J. Imran, N. Narayanan, G. N. Pandian, and B. Raman, "Accurate prediction of covid-19 using chest $\mathrm{x}$-ray images through deep feature learning model with smote and machine learning classifiers," MedRxiv, 2020.

[65] A. Makris, I. Kontopoulos, and K. Tserpes, "Covid-19 detection from chest $\mathrm{x}$-ray images using deep learning and convolutional neural networks," in 11th Hellenic Conference on Artificial Intelligence, pp. 60-66, 2020.

[66] T. Ozturk, M. Talo, E. A. Yildirim, U. B. Baloglu, O. Yildirim, and U. R. Acharya, "Automated detection of covid-19 cases using deep neural networks with x-ray images," Computers in biology and medicine, vol. 121, p. 103792, 2020.

[67] M. Rahimzadeh and A. Attar, “A modified deep convolutional neural network for detecting covid-19 and pneumonia from chest $\mathrm{x}$-ray images based on the concatenation of xception and resnet50v2," Informatics in Medicine Unlocked, vol. 19, p. 100360, 2020.

[68] F. Ucar and D. Korkmaz, “Covidiagnosis-net: Deep bayessqueezenet based diagnosis of the coronavirus disease 2019 (covid-19) from x-ray images," Medical Hypotheses, vol. 140, p. 109761, 2020.

[69] X. Li, C. Li, and D. Zhu, "Covid-mobilexpert: On-device covid19 screening using snapshots of chest $\mathrm{x}$-ray," arXiv preprint arXiv:2004.03042, 2020.

[70] A. I. Khan, J. L. Shah, and M. M. Bhat, "Coronet: A deep neural network for detection and diagnosis of covid-19 from chest $x$-ray images," Computer Methods and Programs in Biomedicine, vol. 196, p. 105581, 2020.

[71] T. Mahmud, M. A. Rahman, and S. A. Fattah, "Covxnet: A multidilation convolutional neural network for automatic covid-19 and other pneumonia detection from chest $x$-ray images with transferable multi-receptive feature optimization," Computers in biology and medicine, vol. 122, p. 103869, 2020.

[72] L. Brunese, F. Mercaldo, A. Reginelli, and A. Santone, "Explainable deep learning for pulmonary disease and coronavirus covid19 detection from x-rays," Computer Methods and Programs in Biomedicine, vol. 196, p. 105608, 2020.

[73] D. Das, K. Santosh, and U. Pal, "Truncated inception net: Covid19 outbreak screening using chest $x$-rays," Physical and engineering sciences in medicine, vol. 43, no. 3, pp. 915-925, 2020.

[74] E. E.-D. Hemdan, M. A. Shouman, and M. E. Karar, "Covidx-net: A framework of deep learning classifiers to diagnose covid-19 in x-ray images," arXiv preprint arXiv:2003.11055, 2020. 
[75] H. Panwar, P. Gupta, M. K. Siddiqui, R. Morales-Menendez, and V. Singh, "Application of deep learning for fast detection of covid-19 in x-rays using ncovnet," Chaos, Solitons \& Fractals, vol. 138, p. 109944, 2020.

[76] D. Singh, V. Kumar, M. Kaur et al., "Classification of covid-19 patients from chest ct images using multi-objective differential evolution-based convolutional neural networks," European Journal of Clinical Microbiology \& Infectious Diseases, vol. 39, no. 7, pp. 1379-1389, 2020.

[77] T. Tuncer, S. Dogan, and F. Ozyurt, "An automated residual exemplar local binary pattern and iterative relieff based covid19 detection method using chest $x$-ray image," Chemometrics and Intelligent Laboratory Systems, vol. 203, p. 104054, 2020.

[78] H. S. Maghdid, A. T. Asaad, K. Z. Ghafoor, A. S. Sadiq, S. Mirjalili, and M. K. Khan, "Diagnosing covid-19 pneumonia from $x$-ray and ct images using deep learning and transfer learning algorithms," in Multimodal Image Exploitation and Learning 2021, vol. 11734, p. 117340E. International Society for Optics and Photonics, 2021.

[79] S. Tian, W. Hu, L. Niu, H. Liu, H. Xu, and S.-Y. Xiao, "Pulmonary pathology of early-phase 2019 novel coronavirus (covid19) pneumonia in two patients with lung cancer," Journal of thoracic oncology, vol. 15, no. 5, pp. 700-704, 2020.

[80] J. Shatri, L. Tafilaj, A. Turkaj, K. Dedushi, M. Shatri, S. Bexheti, and S. K. Mucaj, "The role of chest computed tomography in asymptomatic patients of positive coronavirus disease 2019: a case and literature review," Journal of clinical imaging science, vol. 10, 2020.

[81] Y. Fang, H. Zhang, J. Xie, M. Lin, L. Ying, P. Pang, and W. Ji, "Sensitivity of chest ct for covid-19: comparison to rt-pcr," Radiology, vol. 296, no. 2, pp. E115-E117, 2020.

[82] H. Wang, R. Wei, G. Rao, J. Zhu, and B. Song, "Characteristic ct findings distinguishing 2019 novel coronavirus disease (covid19) from influenza pneumonia," European radiology, vol. 30, no. 9, pp. 4910-4917, 2020.

[83] Y.-n. Duan, Y.-q. Zhu, L.-l. Tang, and J. Qin, "Ct features of novel coronavirus pneumonia (covid-19) in children," European radiology, vol. 30, no. 8, pp. 4427-4433, 2020.

[84] T. Ai, Z. Yang, H. Hou, C. Zhan, C. Chen, W. Lv, Q. Tao, Z. Sun, and L. Xia, "Correlation of chest ct and rt-pcr testing for coronavirus disease 2019 (covid-19) in china: a report of 1014 cases," Radiology, vol. 296, no. 2, pp. E32-E40, 2020.

[85] X. Xie, Z. Zhong, W. Zhao, C. Zheng, F. Wang, and J. Liu, "Chest ct for typical coronavirus disease 2019 (covid-19) pneumonia: relationship to negative rt-pcr testing," Radiology, vol. 296, no. 2, pp. E41-E45, 2020.

[86] S. Hare, A. Tavare, V. Dattani, B. Musaddaq, I. Beal, J. Cleverley, C. Cash, E. Lemoniati, and J. Barnett, "Validation of the british society of thoracic imaging guidelines for covid-19 chest radiograph reporting," Clinical radiology, vol. 75, no. 9, pp. 710-e9, 2020.

[87] J. Lei, J. Li, X. Li, and X. Qi, "Ct imaging of the 2019 novel coronavirus (2019-ncov) pneumonia," Radiology, vol. 295, no. 1, pp. 18-18, 2020.

[88] F. Pan, T. Ye, P. Sun, S. Gui, B. Liang, L. Li, D. Zheng, J. Wang, R. L. Hesketh, L. Yang et al., "Time course of lung changes at chest ct during recovery from coronavirus disease 2019 (covid19)," Radiology, vol. 295, no. 3, pp. 715-721, 2020.

[89] H. Y. F. Wong, H. Y. S. Lam, A. H.-T. Fong, S. T. Leung, T. W.-Y. Chin, C. S. Y. Lo, M. M.-S. Lui, J. C. Y. Lee, K. W.-H. Chiu, T. W.-H. Chung et al., "Frequency and distribution of chest radiographic findings in patients positive for covid-19," Radiology, vol. 296, no. 2, pp. E72-E78, 2020.

[90] H. X. Bai, B. Hsieh, Z. Xiong, K. Halsey, J. W. Choi, T. M. L. Tran, I. Pan, L.-B. Shi, D.-C. Wang, J. Mei et al., "Performance of radiologists in differentiating covid-19 from non-covid-19 viral pneumonia at chest ct," Radiology, vol. 296, no. 2, pp. E46-E54, 2020.

[91] X. Mei, H.-C. Lee, K.-y. Diao, M. Huang, B. Lin, C. Liu, Z. Xie, Y. Ma, P. M. Robson, M. Chung et al., "Artificial intelligenceenabled rapid diagnosis of patients with covid-19," Nature medicine, vol. 26, no. 8, pp. 1224-1228, 2020.

[92] W.-j. Guan, Z.-y. Ni, Y. Hu, W.-h. Liang, C.-q. Ou, J.-x. He, L. Liu, H. Shan, C.-1. Lei, D. S. Hui et al., "Clinical characteristics of coronavirus disease 2019 in china," New England journal of medicine, vol. 382, no. 18, pp. 1708-1720, 2020.
[93] D. Wang, B. Hu, C. Hu, F. Zhu, X. Liu, J. Zhang, B. Wang, H. Xiang, Z. Cheng, Y. Xiong et al., "Clinical characteristics of 138 hospitalized patients with 2019 novel coronavirus-infected pneumonia in wuhan, china," Jama, vol. 323, no. 11, pp. 1061$1069,2020$.

[94] M. Chung, A. Bernheim, X. Mei, N. Zhang, M. Huang, X. Zeng, J. Cui, W. Xu, Y. Yang, Z. A. Fayad et al., "Ct imaging features of 2019 novel coronavirus (2019-ncov)," Radiology, vol. 295, no. 1, pp. 202-207, 2020.

[95] G. D. Rubin, C. J. Ryerson, L. B. Haramati, N. Sverzellati, J. P. Kanne, S. Raoof, N. W. Schluger, A. Volpi, J.-J. Yim, I. B. Martin et al., "The role of chest imaging in patient management during the covid-19 pandemic: a multinational consensus statement from the fleischner society," Chest, vol. 158, no. 1, pp. 106-116, 2020.

[96] S. Rajaraman, J. Siegelman, P. O. Alderson, L. S. Folio, L. R. Folio, and S. K. Antani, "Iteratively pruned deep learning ensembles for covid-19 detection in chest x-rays," IEEE Access, vol. 8, pp. 115041-115050, 2020.

[97] M. Ghaderzadeh and F. Asadi, "Deep learning in detection and diagnosis of covid-19 using radiology modalities: A systematic review," arXiv preprint arXiv:2012.11577, 2020.

[98] S. H. Yoon, K. H. Lee, J. Y. Kim, Y. K. Lee, H. Ko, K. H. Kim, C. M. Park, and Y.-H. Kim, "Chest radiographic and ct findings of the 2019 novel coronavirus disease (covid-19): analysis of nine patients treated in korea," Korean journal of radiology, vol. 21, no. 4, p. $494,2020$.

[99] S. Stephanie, T. Shum, H. Cleveland, S. R. Challa, A. Herring F. L. Jacobson, H. Hatabu, S. C. Byrne, K. Shashi, T. Araki et al., "Determinants of chest x-ray sensitivity for covid-19: A multiinstitutional study in the united states," Radiology: Cardiothoracic Imaging, vol. 2, no. 5, p. e200337, 2020.

[100] R. Yasin and W. Gouda, "Chest x-ray findings monitoring covid19 disease course and severity," Egyptian Journal of Radiology and Nuclear Medicine, vol. 51, no. 1, pp. 1-18, 2020.

[101] M. Zanardo, S. Schiaffino, and F. Sardanelli, "Bringing radiology to patient's home using mobile equipment: A weapon to fight covid-19 pandemic," Clinical Imaging, vol. 68, pp. 99-101, 2020.

[102] M.-Y. Ng, E. Y. Lee, J. Yang, F. Yang, X. Li, H. Wang, M. M.-s. Lui, C. S.-Y. Lo, B. Leung, P.-L. Khong et al., "Imaging profile of the covid-19 infection: radiologic findings and literature review," Radiology: Cardiothoracic Imaging, vol. 2, no. 1, p. e200034, 2020.

[103] I. Castiglioni, D. Ippolito, M. Interlenghi, C. B. Monti, C. Salvatore, S. Schiaffino, A. Polidori, D. Gandola, C. Messa, and F. Sardanelli, "Machine learning applied on chest x-ray can aid in the diagnosis of covid-19: a first experience from lombardy, italy," European Radiology Experimental, vol. 5, no. 1, pp. 1-10, 2021.

[104] X. Yang and C. L. Kovarik, "A systematic review of mobile health interventions in china: identifying gaps in care," Journal of telemedicine and telecare, vol. 27, no. 1, pp. 3-22, 2021.

[105] S. A. H. Tabatabaei, P. Fischer, H. Schneider, U. Koehler, V. Gross, and K. Sohrabi, "Methods for adventitious respiratory sound analyzing applications based on smartphones: A survey," IEEE Reviews in Biomedical Engineering, 2020.

[106] S. Majumder and M. J. Deen, "Smartphone sensors for health monitoring and diagnosis," Sensors, vol. 19, no. 9, p. 2164, 2019.

[107] M. Bauer, T. Glenn, J. Geddes, M. Gitlin, P. Grof, L. V. Kessing, S. Monteith, M. Faurholt-Jepsen, E. Severus, and P. C. Whybrow, "Smartphones in mental health: a critical review of background issues, current status and future concerns," International journal of bipolar disorders, vol. 8, no. 1, pp. 1-19, 2020.

[108] S. Batra, R. A. Baker, T. Wang, F. Forma, F. DiBiasi, and T. PetersStrickland, "Digital health technology for use in patients with serious mental illness: a systematic review of the literature," Medical Devices (Auckland, NZ), vol. 10, p. 237, 2017.

[109] M. Faezipour and A. Abuzneid, "Smartphone-based self-testing of covid-19 using breathing sounds," Telemedicine and e-Health, vol. 26, no. 10, pp. 1202-1205, 2020.

[110] G. Rudraraju, S. Palreddy, B. Mamidgi, N. R. Sripada, Y. P. Sai, N. K. Vodnala, and S. P. Haranath, "Cough sound analysis and objective correlation with spirometry and clinical diagnosis," Informatics in Medicine Unlocked, vol. 19, p. 100319, 2020.

[111] V. Nathan, M. M. Rahman, K. Vatanparvar, E. Nemati, E. Blackstock, and J. Kuang, "Extraction of voice parameters from continuous running speech for pulmonary disease monitoring," in 2019 IEEE International Conference on Bioinformatics and Biomedicine (BIBM), pp. 859-864. IEEE, 2019. 
[112] D. Petrizzo and P. S. Popolo, "Smartphone use in clinical voice recording and acoustic analysis: A literature review," Journal of Voice, 2020.

[113] M. Salathé, C. L. Althaus, R. Neher, S. Stringhini, E. Hodcroft, J. Fellay, M. Zwahlen, G. Senti, M. Battegay, A. Wilder-Smith et al., "Covid-19 epidemic in switzerland: on the importance of testing, contact tracing and isolation," Swiss medical weekly, vol. 150, no. $1112,2020$.

[114] B. Erdogdu Sakar, G. Serbes, and C. O. Sakar, "Analyzing the effectiveness of vocal features in early telediagnosis of parkinson's disease," PloS one, vol. 12, no. 8, p. e0182428, 2017.

[115] L. Brabenec, J. Mekyska, Z. Galaz, and I. Rektorova, "Speech disorders in parkinsonâs disease: early diagnostics and effects of medication and brain stimulation," Journal of neural transmission, vol. 124, no. 3, pp. 303-334, 2017.

[116] E. Maor, J. D. Sara, D. M. Orbelo, L. O. Lerman, Y. Levanon, and A. Lerman, "Voice signal characteristics are independently associated with coronary artery disease," in Mayo Clinic Proceedings, vol. 93, no. 7, pp. 840-847. Elsevier, 2018.

[117] W. H. Organization et al., "Coronavirus disease 2019 (covid-19): situation report, 82," 2020.

[118] A. Imran, I. Posokhova, H. N. Qureshi, U. Masood, M. S. Riaz, K. Ali, C. N. John, M. I. Hussain, and M. Nabeel, “Ai4covid-19: Ai enabled preliminary diagnosis for covid-19 from cough samples via an app," Informatics in Medicine Unlocked, vol. 20, p. 100378, 2020

[119] J. Han, K. Qian, M. Song, Z. Yang, Z. Ren, S. Liu, J. Liu, H. Zheng, W. Ji, T. Koike et al., "An early study on intelligent analysis of speech under covid-19: Severity, sleep quality, fatigue, and anxiety," Proc. Interspeech 2020, pp. 4946-4950, 2020.

[120] Y. Huang, S. Meng, Y. Zhang, S. Wu, Y. Zhang, Y. Zhang, Y. Ye, Q. Wei, N. Zhao, J. Jiang et al., "The respiratory sound features of covid-19 patients fill gaps between clinical data and screening methods," medRxiv, 2020.

[121] B. W. Schuller, D. M. Schuller, K. Qian, J. Liu, H. Zheng, and X. Li, "Covid-19 and computer audition: An overview on what speech \& sound analysis could contribute in the sars-cov-2 corona crisis," arXiv preprint arXiv:2003.11117, 2020.

[122] H. Rohmetra, N. Raghunath, P. Narang, V. Chamola, M. Guizani, and N. R. Lakkaniga, "Ai-enabled remote monitoring of vital signs for covid-19: methods, prospects and challenges," Computing, pp. 1-27, 2021

[123] C. Infante, D. Chamberlain, R. Fletcher, Y. Thorat, and R. Kodgule, "Use of cough sounds for diagnosis and screening of pulmonary disease," in 2017 IEEE Global Humanitarian Technology Conference (GHTC), pp. 1-10. IEEE, 2017.

[124] R. X. A. Pramono, S. A. Imtiaz, and E. Rodriguez-Villegas, "Automatic cough detection in acoustic signal using spectral features," in 2019 41st Annual International Conference of the IEEE Engineering in Medicine and Biology Society (EMBC), pp. 7153-7156. IEEE, 2019

[125] I. D. Miranda, A. H. Diacon, and T. R. Niesler, "A comparative study of features for acoustic cough detection using deep architectures," in 2019 41st Annual International Conference of the IEEE Engineering in Medicine and Biology Society (EMBC), pp. 2601-2605. IEEE, 2019.

[126] M. Soliński, M. Łepek, and Ł. Kołtowski, “Automatic cough detection based on airflow signals for portable spirometry system," Informatics in medicine unlocked, vol. 18, p. 100313, 2020.

[127] C. Menni, A. M. Valdes, M. B. Freidin, C. H. Sudre, L. H. Nguyen, D. A. Drew, S. Ganesh, T. Varsavsky, M. J. Cardoso, J. S. E.-S. Moustafa et al., "Real-time tracking of self-reported symptoms to predict potential covid-19," Nature medicine, vol. 26, no. 7, pp. 1037-1040, 2020.

[128] B. Stasak, Z. Huang, S. Razavi, D. Joachim, and J. Epps, “Automatic detection of covid-19 based on short-duration acoustic smartphone speech analysis," Journal of Healthcare Informatics Research, pp. 1-17, 2021.

[129] J. R. Lechien, C. M. Chiesa-Estomba, P. Cabaraux, Q. Mat, K. Huet, B. Harmegnies, M. Horoi, S. D. Le Bon, A. Rodriguez, D. Dequanter et al., "Features of mild-to-moderate covid-19 patients with dysphonia," Journal of Voice, 2020.

[130] C. Culnane and K. Leins, "Misconceptions in privacy protection and regulation," Law in Context, vol. 36, no. 2, pp. 49-60, 2019.

[131] R. Sun, W. Wang, M. Xue, G. Tyson, S. Camtepe, and D. Ranasinghe, "An empirical assessment of global covid-19 contact tracing applications," 2021.
[132] B. Pranggono and A. Arabo, "Covid-19 pandemic cybersecurity issues," Internet Technology Letters, vol. 4, no. 2, p. e247, 2021.

[133] S. Hakak, W. Z. Khan, M. Imran, K.-K. R. Choo, and M. Shoaib, "Have you been a victim of covid-19-related cyber incidents? survey, taxonomy, and mitigation strategies," IEEE Access, vol. 8, pp. 124 134-124144, 2020.

[134] J. He, L. He, W. Zhou, X. Nie, and M. He, "Discrimination and social exclusion in the outbreak of covid-19," International Journal of Environmental Research and Public Health, vol. 17, no. 8, p. 2933, 2020.

[135] S. H. Alsamhi, B. Lee, M. Guizani, N. Kumar, Y. Qiao, and X. Liu, "Blockchain for decentralized multi-drone to combat covid-19 and future pandemics: Framework and proposed solutions," Transactions on Emerging Telecommunications Technologies, p. e4255.

[136] R. Kumar, A. A. Khan, S. Zhang, W. Wang, Y. Abuidris, W. Amin and J. Kumar, "Blockchain-federated-learning and deep learning models for covid-19 detection using ct imaging," arXiv preprint arXiv:2007.06537, 2020.

[137] M. Torky and A. E. Hassanien, “Covid-19 blockchain framework innovative approach," arXiv preprint arXiv:2004.06081, 2020.

[138] A. Qayyum, K. Ahmad, M. A. Ahsan, A. Al-Fuqaha, and J. Qadir, "Collaborative federated learning for healthcare: Multi-modal covid-19 diagnosis at the edge," arXiv preprint arXiv:2101.07511, 2021

[139] M. A. Rahman, M. S. Hossain, M. S. Islam, N. A. Alrajeh, and G. Muhammad, "Secure and provenance enhanced internet of health things framework: A blockchain managed federated learning approach," Ieee Access, vol. 8, pp. 205 071-205 087, 2020.

[140] A. Ulhaq and O. Burmeister, "Covid-19 imaging data privacy by federated learning design: A theoretical framework," arXiv preprint arXiv:2010.06177, 2020.

[141] Q. Yang, J. Zhang, W. Hao, G. Spell, and L. Carin, “Flop: Federated learning on medical datasets using partial networks," arXiv preprint arXiv:2102.05218, 2021.

[142] P. Wang, C. Lin, M. S. Obaidat, Z. Yu, Z. Wei, and Q. Zhang, "Contact tracing incentive for covid-19 and other pandemic diseases from a crowdsourcing perspective," IEEE Internet of Things Journal, 2021.

[143] A. Alimadadi, S. Aryal, I. Manandhar, P. B. Munroe, B. Joe, and $X$. Cheng, "Artificial intelligence and machine learning to fight covid-19," 2020.

[144] M. Loey, F. Smarandache, and N. E. M Khalifa, "Within the lack of chest covid-19 x-ray dataset: a novel detection model based on gan and deep transfer learning," Symmetry, vol. 12, no. 4, p. 651, 2020.

[145] M.-H. Tayarani-N, "Applications of artificial intelligence in battling against covid-19: a literature review," Chaos, Solitons $\mathcal{E}$ Fractals, p. 110338, 2020.

[146] T. F. Quatieri, T. Talkar, and J. S. Palmer, "A framework for biomarkers of covid-19 based on coordination of speechproduction subsystems," IEEE Open Journal of Engineering in Medicine and Biology, vol. 1, pp. 203-206, 2020.

[147] G. Pinkas, Y. Karny, A. Malachi, G. Barkai, G. Bachar, and V. Aharonson, "Sars-cov-2 detection from voice," IEEE Open Journal of Engineering in Medicine and Biology, vol. 1, pp. 268-274, 2020.

[148] J. M. Purswani, A. P. Dicker, C. E. Champ, M. Cantor, and N. Ohri, "Big data from small devices: the future of smartphones in oncology," in Seminars in radiation oncology, vol. 29, no. 4, pp. 338-347. Elsevier, 2019.

[149] P. Silva, E. Luz, G. Moreira, C. Gomes, L. Viana, and R. Silva, "Pictures of $x$-rays displayed in monitors for deep learningbased covid-19 screening: Implications for mobile application development," 2020.

[150] T. Greenhalgh, J. Wherton, S. Shaw, and C. Morrison, "Video consultations for covid-19," The BMJ, vol. 368, 2020.

[151] J. E. Hollander and B. G. Carr, "Virtually perfect? telemedicine for covid-19," New England Journal of Medicine, vol. 382, no. 18, pp. $1679-1681,2020$

[152] E. M. Abou-Nassar, A. M. Iliyasu, P. M. El-Kafrawy, O.-Y. Song, A. K. Bashir, and A. A. Abd El-Latif, "Ditrust chain: towards blockchain-based trust models for sustainable healthcare iot systems," IEEE Access, vol. 8, pp. 111 223-111 238, 2020.

[153] R. Vaishya, M. Javaid, I. H. Khan, and A. Haleem, "Artificial intelligence (ai) applications for covid-19 pandemic," Diabetes $\mathcal{E}$ Metabolic Syndrome: Clinical Research \& Reviews, vol. 14, no. 4, pp. 337-339, 2020. 
[154] M. Nguyen, M. Waller, A. Pandya, and J. Portnoy, "A review of patient and provider satisfaction with telemedicine," Current allergy and asthma reports, vol. 20, no. 11, pp. 1-7, 2020.

[155] T. C. O. Hashiguchi, "Bringing health care to the patient: An overview of the use of telemedicine in oecd countries," OECD Health Working Papers, no. 116, pp. 0_1-101, 2020.

[156] A. C. Smith, E. Thomas, C. L. Snoswell, H. Haydon, A. Mehrotra, J. Clemensen, and L. J. Caffery, "Telehealth for global emergencies: Implications for coronavirus disease 2019 (covid-19)," Journal of telemedicine and telecare, vol. 26, no. 5, pp. 309-313, 2020.

[157] X. Zhou, C. L. Snoswell, L. E. Harding, M. Bambling, S. Edirippulige, X. Bai, and A. C. Smith, "The role of telehealth in reducing the mental health burden from covid-19," Telemedicine and $e^{-}$ Health, vol. 26, no. 4, pp. 377-379, 2020.

[158] J. Thirthalli, N. Manjunatha, and S. B. Math, "Unmask the mind! importance of video consultations in psychiatry during covid-19 pandemic," Schizophrenia research, 2020.

[159] A. M. Morenz, S. Wescott, A. Mostaghimi, T. D. Sequist, and M. Tobey, "Evaluation of barriers to telehealth programs and dermatological care for american indian individuals in rural communities," JAMA dermatology, vol. 155, no. 8, pp. 899-905, 2019.

[160] E. Monaghesh and A. Hajizadeh, "The role of telehealth during covid-19 outbreak: a systematic review based on current evidence," BMC Public Health, vol. 20, no. 1, pp. 1-9, 2020.

[161] J. Portnoy, M. Waller, and T. Elliott, "Telemedicine in the era of covid-19," The Journal of Allergy and Clinical Immunology: In Practice, vol. 8, no. 5, pp. 1489-1491, 2020.

[162] C. L. Fleischhacker, "Patient satisfaction with telehealth services compared to in-office visits: A systematic literature review," 2020.

[163] A. A. Nasser, R. M. Alzahrani, C. A. Fellah, D. M. Jreash, N. T. A. Almuwallad, D. S. A. Bakulka, and R. A. R. Abed, "Measuring the patientsâ satisfaction about telemedicine used in saudi arabia during covid-19 pandemic," Cureus, vol. 13, no. 2, 2021.

[164] R. Bashshur, C. R. Doarn, J. M. Frenk, J. C. Kvedar, and J. O. Woolliscroft, "Telemedicine and the covid-19 pandemic, lessons for the future," 2020.

[165] Q.-V. Pham, D. C. Nguyen, T. Huynh-The, W.-J. Hwang, and P. N. Pathirana, "Artificial intelligence (ai) and big data for coronavirus (covid-19) pandemic: A survey on the state-of-thearts," 2020.

[166] A. L. McGuire, M. P. Aulisio, F. D. Davis, C. Erwin, T. D. Harter, R. Jagsi, R. Klitzman, R. Macauley, E. Racine, S. M. Wolf et al., "Ethical challenges arising in the covid-19 pandemic: An overview from the association of bioethics program directors (abpd) task force," The American Journal of Bioethics, vol. 20, no. 7, pp. 15-27, 2020.

[167] K. Dalkir and R. Katz, Navigating Fake News, Alternative Facts, and Misinformation in a Post-truth World. IGI Global, 2020.

[168] N. L. Kolluri and D. Murthy, "Coverifi: A covid-19 news verification system," Online Social Networks and Media, vol. 22, p. 100123, 2021.

[169] S. Shifath, M. F. Khan, M. Islam et al., "A transformer based approach for fighting covid-19 fake news," arXiv preprint arXiv:2101.12027, 2021.

[170] C. M. Pulido, B. Villarejo-Carballido, G. Redondo-Sama, and A. Gómez, "Covid-19 infodemic: More retweets for sciencebased information on coronavirus than for false information," International Sociology, vol. 35, no. 4, pp. 377-392, 2020.

[171] R. F. Sear, N. Velasquez, R. Leahy, N. J. Restrepo, S. El Oud, N. Gabriel, Y. Lupu, and N. F. Johnson, "Quantifying covid19 content in the online health opinion war using machine learning," Ieee Access, vol. 8, pp. 91 886-91 893, 2020.

[172] N. Puri, E. A. Coomes, H. Haghbayan, and K. Gunaratne, "Social media and vaccine hesitancy: new updates for the era of covid-19 and globalized infectious diseases," Human Vaccines $\mathcal{E}$ Immunotherapeutics, pp. 1-8, 2020.

[173] J. V. Lazarus, S. C. Ratzan, A. Palayew, L. O. Gostin, H. J. Larson, K. Rabin, S. Kimball, and A. El-Mohandes, "A global survey of potential acceptance of a covid-19 vaccine," Nature medicine, vol. 27, no. 2, pp. 225-228, 2021.

[174] I. Montagni, K. Ouazzani-Touhami, A. Mebarki, N. Texier, S. Schück, C. Tzourio et al., "Acceptance of a covid-19 vaccine is associated with ability to detect fake news and health literacy," Journal of public health (Oxford, England), 2021.

[175] N. Noë, R. M. Whitaker, M. J. Chorley, and T. V. Pollet, "Birds of a feather locate together? foursquare checkins and personality homophily," Computers in Human Behavior, vol. 58, pp. 343-353, 2016.

[176] D. M. Lazer, M. A. Baum, Y. Benkler, A. J. Berinsky, K. M. Greenhill, F. Menczer, M. J. Metzger, B. Nyhan, G. Pennycook, D. Rothschild et al., "The science of fake news," Science, vol. 359, no. 6380, pp. 1094-1096, 2018.

[177] A. N. M. Yusof, M. Z. Muuti, L. A. Ariffin, and M. K. M. Tan, "Sharing information on covid-19: the ethical challenges in the malaysian setting," Asian bioethics review, vol. 12, no. 3, pp. 349361, 2020.

[178] C. Ireton and J. Posetti, Journalism, fake news $\mathcal{E}$ disinformation: handbook for journalism education and training. Unesco Publishing, 2018.

[179] I. Ahmad, M. Yousaf, S. Yousaf, and M. O. Ahmad, "Fake news detection using machine learning ensemble methods," Complexity, vol. 2020, 2020.

[180] W. S. Paka, R. Bansal, A. Kaushik, S. Sengupta, and T. Chakraborty, "Cross-sean: A cross-stitch semi-supervised neural attention model for covid-19 fake news detection," arXiv preprint arXiv:2102.08924, 2021.

[181] S. Gundapu and R. Mamid, "Transformer based automatic covid19 fake news detection system," arXiv preprint arXiv:2101.00180, 2021.

[182] F. A. Ozbay and B. Alatas, "Fake news detection within online social media using supervised artificial intelligence algorithms," Physica A: Statistical Mechanics and its Applications, vol. 540, p. $123174,2020$.

[183] B. Chen, B. Chen, D. Gao, Q. Chen, C. Huo, X. Meng, W. Ren, and Y. Zhou, "Transformer-based language model fine-tuning methods for covid-19 fake news detection," arXiv preprint arXiv:2101.05509, 2021.

[184] G. D. Smith, F. Ng, and W. H. C. Li, "Covid-19: Emerging compassion, courage and resilience in the face of misinformation and adversity," Journal of clinical nursing, vol. 29, no. 9-10, p. 1425, 2020.

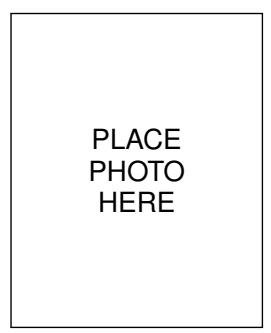

Q uratulain Arshad received her BS in Computer Science from Comsats Institute of Information Technology, Pakistan. She is currently pursuing her Master Degree (Computer Science) from Comsat University Islamabad, Wah Campus. Her research interests include network security, trust and reputation in sensor networks and ad hoc networks. Her subjects of interest include Network Security, Machine Learning Deep Learning, Digital Image Processing and Computer Vision.

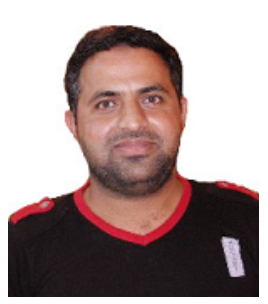

Wazir Zada Khan (M'16-SM'17) is currently working as an Associate Professor at Department of Computer Science, Capital University of Science and Technology, Islamabad, Pakistan. Dr. Khan is also serving as a Researcher at the 'Global Foundation for Cyber Studies and Research' (https://www.gfcyber.org), which is an independent, non-profit, and non-partisan cybersecurity think-tank based in Washington D.C. Dr. Khan received his bachelor's and master's degrees in computer science from COMSATS

University Islamabad, Wah Campus in 2004 and 2007, respectively. $\mathrm{He}$ received his $\mathrm{PhD}$ from the Electrical and Electronic Engineering Department, Universiti Teknologi PETRONAS, Malaysia in 2015. Dr. Khan has published over 75 research papers in the journals and conferences of international repute. He is the serving as a reviewer of many reputed journals and also a member of the technical program committee for many international conferences. He has more than ten years of teaching/professional experience in Pakistan and Saudi Arabia. His current research interests include wireless sensor networks, security and privacy, blockchain, IoT, IloT and reinforcement learning. He is a Senior Member of the IEEE. 


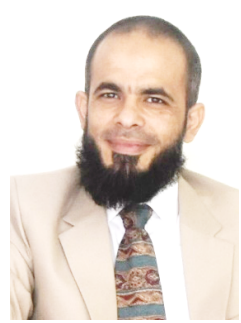

Faisal Azam is working as an Assistant Professor, COMSATS University Islamabad, Pakistan. $\mathrm{He}$ is a PhD with interests in artificial intelligence, data sciences, image processing and machine learning techniques. He has vast research experience in seismic intelligent data modeling, agricultural prediction, medical image analysis and other related fields. His core competencies are multi agent modeling and evolutionary algorithm applications including swarm intelligence and genetic algorithms. He has completed various undergraduate fully funded projects in artificial intelligence domain. Currently he completed product-oriented work on cognitive management software support for UAV devices funded by a government agency. His undergoing research work is related to decision support system for Blockchain platforms.

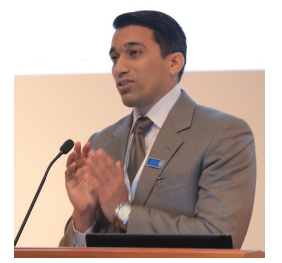

Muhammad Khurram Khan is currently working as a Professor of Cybersecurity at the Center of Excellence in Information Assurance, King Saud University, Kingdom of Saudi Arabia. $\mathrm{He}$ is founder and CEO of the 'Global Foundation for Cyber Studies and Research' (http://www.gfcyber.org), an independent and non-partisan cybersecurity think-tank in Washington D.C, USA. He is the Editor-in-Chief of 'Telecommunication Systems' published by Springer-Nature with its recent impact factor of 1.73 (JCR 2020). He is on the editorial board of several journals including, IEEE Communications Surveys \& Tutorials, IEEE Communications Magazine, IEEE Internet of Things Journal, IEEE Transactions on Consumer Electronics, Journal of Network \& Computer Applications (Elsevier), IEEE Access, IEEE Consumer Electronics Magazine, PLOS ONE, and Electronic Commerce Research, etc. He has published more than 400 papers in the journals and conferences of international repute. In addition, he is an inventor of 10 US/PCT patents. He has edited 10 books/proceedings published by Springer-Verlag, Taylor \& Francis and IEEE. His research areas of interest are Cybersecurity, digital authentication, loT security, biometrics, multimedia security, cloud computing security, cyber policy, and technological innovation management. He is a fellow of the IET (UK), a fellow of the BCS (UK), and a fellow of the FTRA (Korea). He is the Vice Chair of IEEE Communications Society Saudi Chapter. He is a distinguished Lecturer of the IEEE. His detailed profile can be visited at http://www.professorkhurram.com. 